Portland State University

PDXScholar

Fall 12-4-2017

\title{
Physics Based Approach for Seafloor Classification
}

Phu Duy Nguyen

Portland State University

Follow this and additional works at: https://pdxscholar.library.pdx.edu/open_access_etds

Part of the Electrical and Computer Engineering Commons Let us know how access to this document benefits you.

\section{Recommended Citation}

Nguyen, Phu Duy, "Physics Based Approach for Seafloor Classification" (2017). Dissertations and Theses. Paper 4060.

https://doi.org/10.15760/etd.5944

This Thesis is brought to you for free and open access. It has been accepted for inclusion in Dissertations and Theses by an authorized administrator of PDXScholar. Please contact us if we can make this document more accessible: pdxscholar@pdx.edu. 
Physics Based Approach for Seafloor Classification

\title{
by
}

\section{Phu Duy Nguyen}

\section{A thesis submitted in partial fulfillment of the} requirements for the degree of

\author{
Master of Science \\ in \\ Electrical and Computer Engineering
}

Thesis Committee:

Martin Siderius, Chair

Branimir Pejcinovic

Jonathan Bird

Portland State University

2017 


\begin{abstract}
The seafloor properties are of high importance for many applications such as marine biology, oil and gas exploration, laying cables, dredging operations and off-shore construction. Several approaches exist to classify the properties of the seabed. These include taking direct samples of the seabed (e.g., coring), however, these methods are are costly and slow. Underwater acoustic remote sensing techniques are of interest because they are lower cost and faster. The information about the seabed properties can be extracted by studying the energy of single beam echo sounders (SBES). This can be done by either phenomenological or numerical methods [1], [2]. This research investigates a numerical, model-data fitting method using a high frequency backscattering model developed by Jackson et al [3]. In this "inversion modeling" method, the matching process between the model and average echo envelope provides information about the sediment parameters, namely the sediment mean grain size $\left(M_{z}\right)$ as the indicator of the seabed type, spectral parameter $\left(W_{2}\right)$ as the indicator of seabed roughness and normalized sediment volume parameter $\sigma_{2}$ as the indicator of the scattering due to sediment inhomogeneities.
\end{abstract}




\section{Acknowledgements}

I would like to thank the people who provided support and encouragement throughout my studies in last two years and my final project. Without their support I would not be able to complete my thesis.

I would like first to thank my advisor Dr. Martin Siderius for his continuous support, patience to guide me throughout the whole project. He was always the first resource I came to whenever I need help on my studies and research.

I would also like to acknowledge Dr. Branimir Pejcinovic and Dr. Jonathan Bird for their serving on my thesis committee.

I am also grateful to all people at Northwest Electromagnetics and Acoustics Research Laboratory (NEAR Lab) at Portland State University and Metron Scientific Solution for their help and support. Especially, I would like to thank Dr. Elizabeth Kusel from NEAR Lab, who was always ready to accompany with me on any field experiments that I need for my project.

I also would like to thank Dr. D.J Tang and Dr. Todd Hefner from Applied Physics Laboratory at University of Washington who has supported me by providing his experimental data for my project.

Appreciation goes out to my family, my friends for their patience, understanding during my study. Without their moral support, I would not be able to achieve my goal. 


\section{Contents}

Abstract i

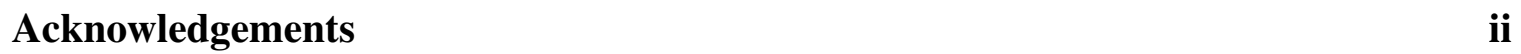

List of Tables $\quad$ vi

\begin{tabular}{ll}
\hline List of Figures & 1
\end{tabular}

$\begin{array}{lll}1 & \text { Introduction } & 1\end{array}$

\begin{tabular}{|lll}
2 & Background & 3
\end{tabular}

2.1 Fundamental concept and definition . . . . . . . . . . . . . . . 3

2.1 .1 Sound waves in medium . . . . . . . . . . . . . 3

2.1 .2 Wavefronts . . . . . . . . . . . . . . . 4

2.1 .3 Sound pressure . . . . . . . . . . . . . . 5

2.1 .4 Speed of sound . . . . . . . . . . . . . . . 6

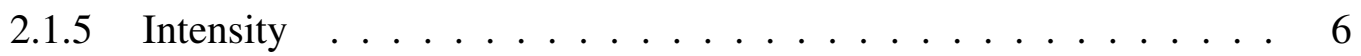

2.1 .6 Characteristic Impedance . . . . . . . . . . . . . . . . . 6

2.1 .7 Wave equation . . . . . . . . . . . . . 7

2.1 .8 Transmission loss . . . . . . . . . . . . . . . . . . . 8

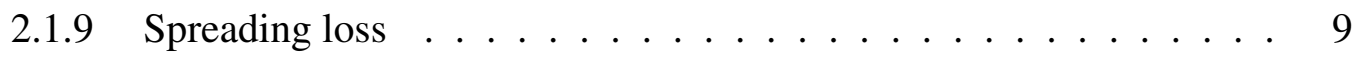

2.1 .10 Absorption loss . . . . . . . . . . . . . . . 10

2.2 Reflection and scattering . . . . . . . . . . . . . . 11

2.2 .1 Reflection . . . . . . . . . . . . . . . 11

2.2 .2 Scattering . . . . . . . . . . . . . . . . 14

3 Underwater acoustic systems and empirical approach for seabed classification 19

3.1 Acoustic instrument . . . . . . . . . . . . . . . . . . . . . . . . 19 
$3.1 .1 \quad$ Single-beam echosounder (SBES) . . . . . . . . . . . . . . . . . 19

3.1 .2 Sidescan sonar $(\mathrm{SSS}) \ldots \ldots \ldots \ldots \ldots$

3.1 .3 Multi-row SSS . . . . . . . . . . . . . . . . . . 21

3.1 .4 Multibeam sonar (MBES) $\ldots \ldots \ldots \ldots \ldots \ldots$

3.2 Phenomenological approach using SBES for seabed classification . . . . 22

3.2 .1 The multi-echo energy approach of RoxAnn system . . . . . . . 22

3.2 .2 Quester Tangent Corporation (QTC) approach. . . . . . . . . . . 24

$\begin{array}{|lll|}4 & \text { Theoretical model and methodology for model-based seabed classification } 25\end{array}$

4.1 Theoretical model . . . . . . . . . . . . . . . . . . . . . . 25

4.1 .1 Model Inputs: Sea floor parameters … . . . . . . . . 25

4.1 .2 Model for near vertical backscattering due to roughness . . . . . 26

4.1 .3 Volume scattering model . . . . . . . . . . . . . . . . . . 32

4.2 Optimization methodology $\ldots \ldots \ldots \ldots$

4.2 .1 Model function . . . . . . . . . . . . . . . . . . . 35

4.2 .2 Cost function $\ldots \ldots \ldots \ldots \ldots \ldots \ldots$

4.2 .3 Search Algorithm $\ldots \ldots \ldots \ldots$. . . . . . . . . . . 41

4.2 .4 Testing the optimization with synthetic data $\ldots \ldots \ldots \ldots$

5 Applying the model to measured data 49

5.1 Data processing . . . . . . . . . . . . . . . . . . . . . . . . . 49

$5.1 .1 \quad$ Correcting the signal amplitude $\ldots \ldots \ldots$. . . . . . . 49

5.1 .2 Normalizing the data $\ldots \ldots \ldots \ldots \ldots \ldots$

5.1 .3 Aligning the signal $\ldots \ldots \ldots \ldots \ldots \ldots \ldots$

5.1 .4 Averaging the signal $\ldots \ldots \ldots \ldots \ldots \ldots \ldots \ldots$

5.2 Target and Reverberation Experiment 2013 (TREX13) data . . . . . . . 53

$5.2 .1 \quad$ Description of data set $\ldots \ldots \ldots \ldots \ldots$

5.2 .2 Optimization results $\ldots \ldots \ldots \ldots \ldots \ldots$

5.3 Mapping with Waldo Lake data $\ldots \ldots \ldots \ldots$ 
$5.3 .1 \quad$ Description of data set $\ldots . \ldots . \ldots 59$

5.3 .2 Optimization result: . . . . . . . . . . . . . 59

$\begin{array}{llr}6 & \text { Discussion } & 65\end{array}$

\begin{tabular}{ll}
\hline Bibliography & 68
\end{tabular}

\begin{tabular}{|ll|}
\hline Appendix A & APL model parameterizations
\end{tabular}

\begin{tabular}{|ll|}
\hline Appendix B & The roughness scattering approximation \\
\hline
\end{tabular}

\begin{tabular}{|ll|}
\hline Appendix C & The volume scattering approximation \\
\hline
\end{tabular} 


\section{List of Tables}

4.1 Parameterization of inputs in term of bulk mean grain size $M_{z}$ (defined in

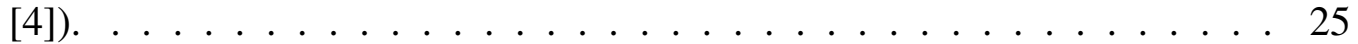

4.2 Summary of test with synthetic data $\ldots \ldots \ldots$. . . . . . . . . . . 48

$5.1 \quad$ Summary of optimization results with measured data. . . . . . . . . . . 63

5.2 Empirical data listed in [4]. . . . . . . . . . . . . . . . . 63 


\section{List of Figures}

2.1 Graphical representation of sound wave in water. The figure is adapted from $[5] . \ldots \ldots \ldots \ldots$

2.2 In far field, at a long distance from source, the spherical wavefront (a) looks like plane wave (b). . . . . . . . . . . . . . . . . . . 5

2.3 Geometry for illustrating angular coordinates used in treating reflections and scattering (adapted from [3]). $\ldots \ldots \ldots$

2.4 High level schematic to describe acoustic scattering due to the roughness of water-sediment interface and heterogeneity of the sediment (adapted from

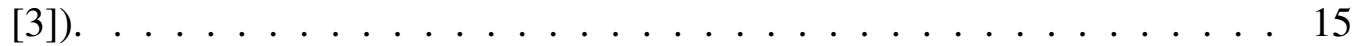

3.1 An illustration of SBES . The figure is adapted from [6]. . . . . . . . . . . 20

3.2 Simple sidescan consists of single beam echosounder per side . The figure

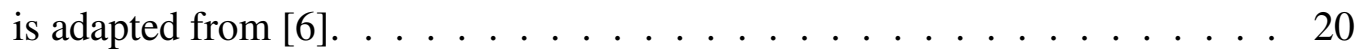

3.3 Multiple row sidescan consists of multiple single beam echosounder per

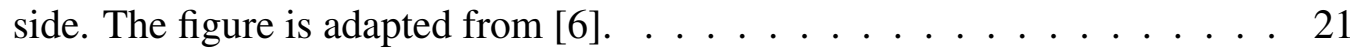

3.4 The multibeam sonar head is arranged in a Mills Cross with an array of transmitters and array of beam steered hydrophones. This figure is adapted from $[6].] \ldots \ldots \ldots \ldots . \ldots \ldots$

3.5 Illustration of working principle of RoxAnn system. This figure is adapted from $[6] . \ldots \ldots \ldots \ldots . \ldots \ldots \ldots$

$4.1 \quad$ Illustration of interface scattering geometry. . . . . . . . . . . . . 27

4.2 An example of signal footprint for $t_{0}<t<t_{0}+\tau$. . . . . . . 30

4.3 Illustration of volume scattering geometry . . . . . . . . . . . . 33

4.4 Modeled signal with rectangular pulse. . . . . . . . . . . . . . . . 36

$4.5 \quad$ Modeled signal different interface and volume scattering parameters. . . . . 37 
4.6 Modeled signal with Gaussian window. $\ldots \ldots \ldots \ldots \ldots$

4.7 Best fit model vs. synthetic data. . . . . . . . . . . . . . . . . 44

4.8 Estimated mean grain size $M_{z} . \ldots \ldots \ldots \ldots \ldots \ldots$

$4.9 \quad$ Estimated spectral strength $W_{2} . \ldots \ldots \ldots \ldots \ldots$

4.10 Density ratio $\rho$. True value is $1.5176 . \ldots \ldots \ldots \ldots$. . . . . . 46

4.11 Speed ratio $v$. True value is 1.1660 . . . . . . . . . . . . . 46

4.12 Loss parameter $\delta$. True value is 0.0183 . . . . . . . . . . . 47

4.13 Interface scattering cross section $\sigma_{s}$. True value is $0.2097 \ldots \ldots \ldots$

4.14 Volume scattering parameter $\sigma_{2}$. True value is 0.0021 . . . . . . . . 48

$5.1 \quad$ Acoustic data collected by NEAR lab at Waldo lake, Sept 2017 . . . . . . 50

5.2 Diagram of multipaths data $\ldots \ldots \ldots \ldots \ldots \ldots \ldots \ldots$

5.3 Acoustic data collected by Applied Phisycs Laboratory, University of Wash-

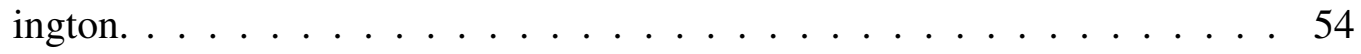

5.4 Model vs. measured data $\ldots \ldots \ldots \ldots$. . . . . . . . . . 54

$5.5 \quad$ Estimated mean grain size $M_{z} . \ldots \ldots \ldots \ldots \ldots \ldots$

$5.6 \quad$ Estimated spectral strength $W_{2} . \ldots \ldots \ldots \ldots \ldots \ldots$

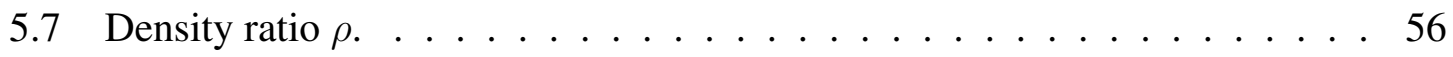

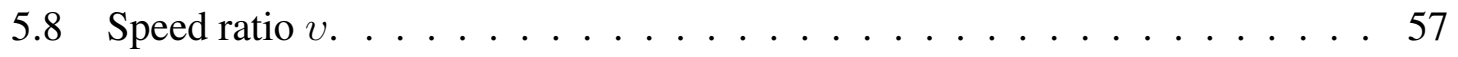

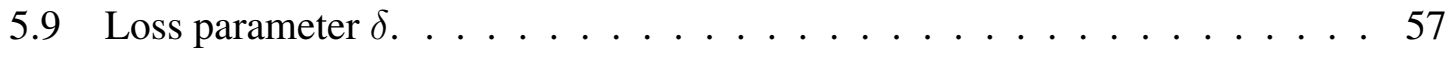

5.10 Interface scattering cross section $\sigma_{s} . \ldots \ldots \ldots \ldots \ldots$

5.11 Volume scattering parameter $\sigma_{2} . \ldots \ldots \ldots \ldots \ldots \ldots$

5.12 Estimated mean grain size $M_{z} . \ldots \ldots \ldots \ldots \ldots \ldots$

5.13 Estimated spectral strength $W_{2} . \ldots \ldots \ldots \ldots$

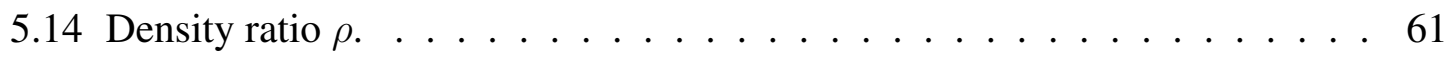

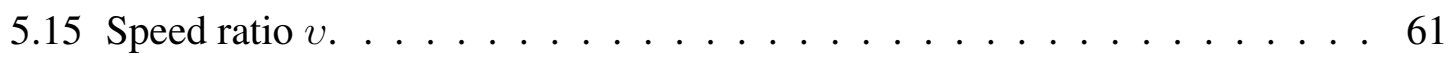

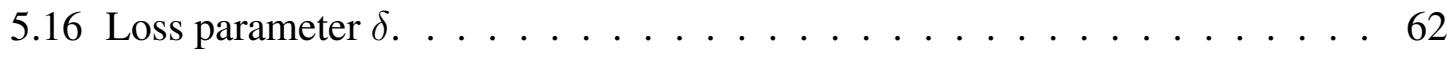

5.17 Interface scattering cross section $\sigma_{s} . \ldots \ldots \ldots \ldots \ldots \ldots \ldots \ldots$

5.18 Volume scattering parameter $\sigma_{2} . \ldots \ldots \ldots \ldots \ldots \ldots \ldots \ldots$ 


\section{Chapter 1}

\section{Introduction}

Recently, a variety of alternative technologies for mapping seabed habitats, such as side-scan sonar, underwater cameras, sediment profiling imagery (SPI), satellite images, as well as acoustic seabed classification systems [6] have become more common [7]. Some of them require ground truth for calibration, while others do not. In this report a physics based method is described to estimate seabed properties from normal incident acoustic echoes with a single beam echosounder system (SBES). This is a remote sensing approach and does not require direct sampling of the seabed. The method uses data fitting of a theoretical model parameterized by seabed properties. The theoretical model simulates the shape of the echo return based on the environmental inputs which are adjusted to optimize the fit to the acoustic measurements. The best match of this process is expected to give the approximate sediment parameters such as scattering cross section, volume and loss parameters, sediment-water density ratio, sediment water sound speed ratio, mean grain size and spectral strength. These quantities will be described in more detail in section 2 .

The optimization process involves two steps. First, the normal incidence bottom reflection coefficient of each ping is estimated from acoustic echo data and an empirical relationship between reflection coefficient and sediment mean grain size is employed [8]. The mean grain size is then used to infer the range of possible values for each input of the 
model (based on empirical relationships [4]). This approach reduces computational cost and constrains the searching results to be physically meaningful. The second step compares the theoretical echo envelope with the measurement and searches over all possible parameter values to determine the set that minimizes the difference between the modeled and measured signals. The theoretical model and optimization methodology are described in section 4 . Section 5 presents the results of model fitting to measured data. Section 6 gives a discussion about the results. 


\section{Chapter 2}

\section{Background}

\subsection{Fundamental concept and definition}

\subsubsection{Sound waves in medium}

A wave motion, in which the particles of the medium oscillate about their mean positions in the direction of propagation of the wave, is called a longitudinal wave. Sound waves are classified as longitudinal waves. A sound wave propagating underwater consists of alternating compression and rarefaction [9] of the medium as shown in Fig.2.1.

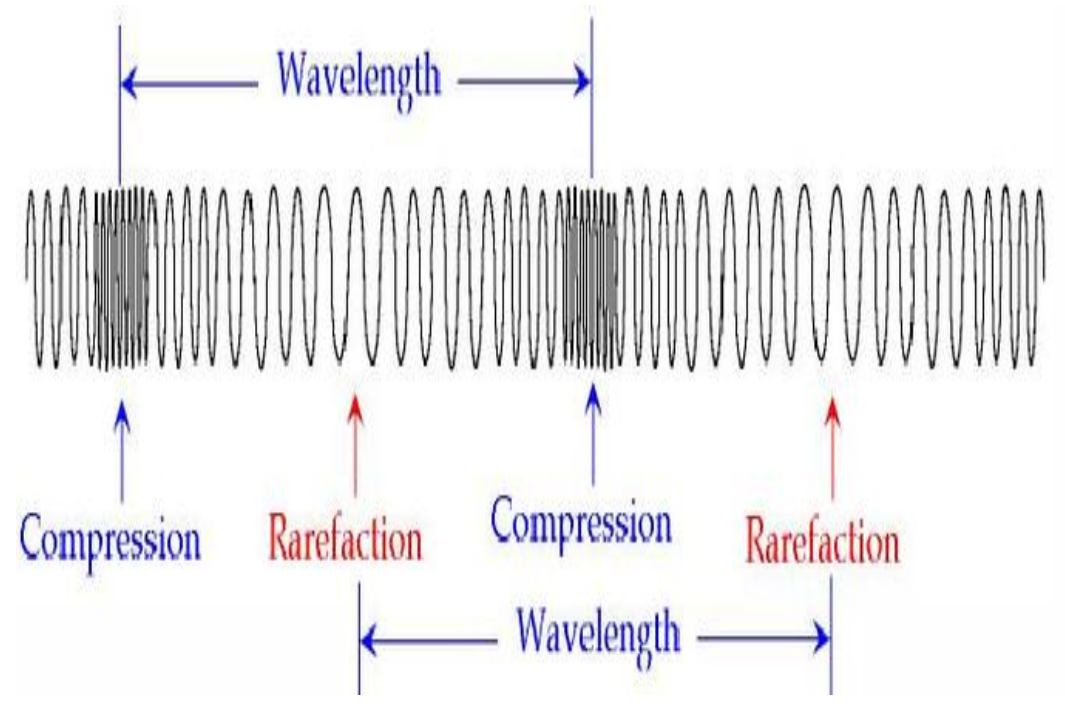

Figure 2.1: Graphical representation of sound wave in water. The figure is adapted from [5]. 


\subsubsection{Wavefronts}

The form of any wave is determined by its source and is described by the shape of the wavefronts. There are three basic types of waves, plane, spherical and cylindrical. A plane wave is emitted by a planar source, a cylindrical by line source and spherical by point source. Plane waves are not really possible to create in reality but it is a useful approximation [10] as at a long distance spherical waves look like a plane wave. Figure 2.1 illustrates spherical and plane wavefronts. In a $2 \mathrm{D}$ plot, the cross section of a spherical wave is shown in Fig. 2.2a, and the same wave at long distance is shown in Fig. 2.2b showing an example of the spherical wave appearing as a plane wave structure at long distances from the point source. 


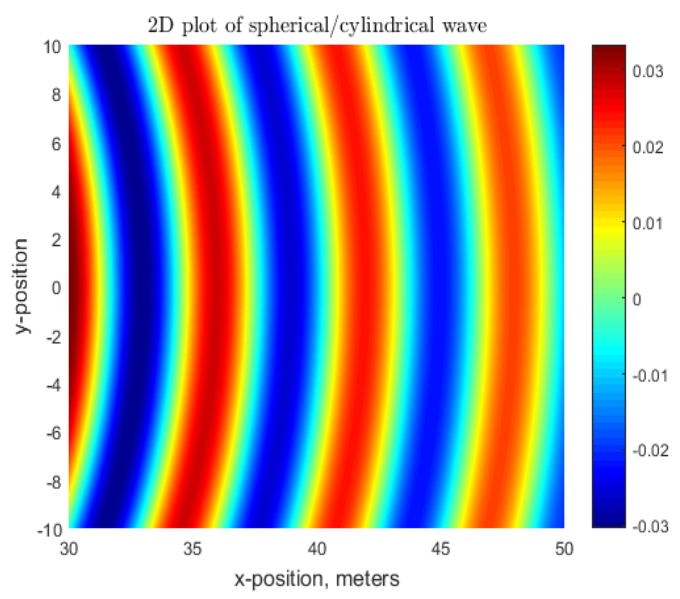

(a)

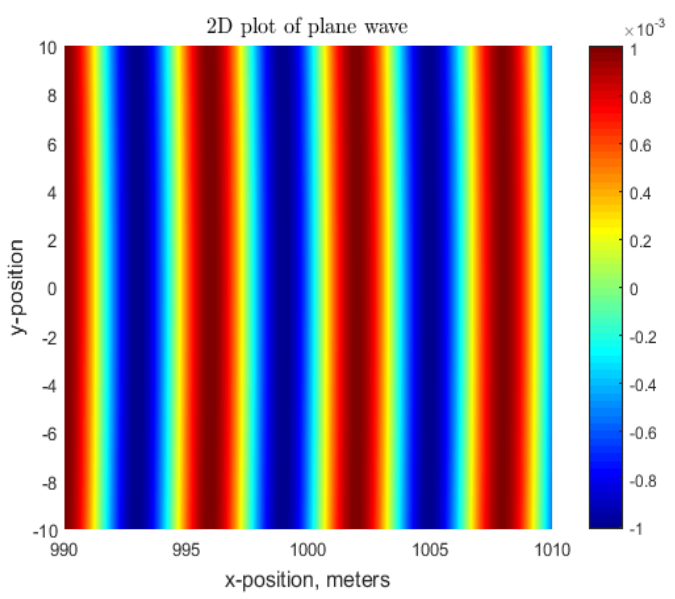

(b)

Figure 2.2: In far field, at a long distance from source, the spherical wavefront (a) looks like plane wave (b).

\subsubsection{Sound pressure}

The sound pressure is the force of sound on a surface area perpendicular to the direction of the sound. The sound pressure can also sometimes be called acoustic pressure. The standardized unit for acoustic pressure is Pascals $(\mathrm{Pa})$. The sound pressure in air can be measured with microphone and underwater acoustic pressure can be measured by hydrophone. 


\subsubsection{Speed of sound}

Three acoustic quantities such as speed of sound $c_{w}$ (i.e., the longitudinal motion of wave), sound frequency $f$ and wavelength known as $\lambda$ are related by

$$
f=\frac{c_{w}}{\lambda} .
$$

\subsubsection{Intensity}

A propagating sound wave carries mechanical energy with it in the form of kinetic energy of the particles in motion plus the potential energy of the stresses set up in the elastic medium. Because the wave is propagating, a certain amount of energy per second, or power, is crossing a unit area and this power per unit area, or power density is called the intensity, $I$, of the wave. In water, the intensity $I$ is proportional to the square of the acoustic pressure $p$ by

$$
I=\frac{p^{2}}{\rho_{m} c_{m}}
$$

where $\rho_{m}$ and $c_{m}$ are medium density and speed of sound in the medium.

\subsubsection{Characteristic Impedance}

The product of two term $c_{m}$ and $\rho_{m}$ is called the characteristic impedance of the media. It is property of a sound medium that is analogous to the impedance in electrical circuit theory. 


\subsubsection{Wave equation}

The sound propagates in the medium at the speed $c_{m}$ (or $c_{w}$ for water). The propagation of the sound can be described mathematically by solution of wave equation,

$$
\frac{d^{2} p}{d t^{2}}=c^{2}\left(\frac{d^{2} p}{d x^{2}}+\frac{d^{p}}{d y^{2}}+\frac{d^{2} p}{d z^{2}}\right)
$$

which can also be written,

$$
\nabla^{2} p=\frac{1}{c^{2}} \frac{d^{2} p}{d t^{2}}
$$

The wave equation is written in a partial differential form in term of acoustic pressure $p$ with respect to the coordinates $x, y, z$ and time $t$. For one-dimension, all functions $p(x, t)$ that fulfill equation 2.4 have the form:

$$
p(x, t)=f(c t \pm x)
$$

If the sound wave was a sinusoidal oscillation (i.e., time-harmnic solution), for different wave types the complex representation of the sound pressure (in units of Pascal) is written as follows, for plane waves,

$$
p(x, t)=p_{0} e^{i( \pm k x+\phi)} e^{i \omega t}
$$

where the \pm is + for waves propagating in the negative $x$ direction and is - for waves 
propagating in the positive $x$ direction. And, for outgoing spherical waves:

$$
p(r, t)=\frac{p_{0}}{r} e^{i(-k r+\phi)} e^{i \omega t}
$$

where $\phi$ is a phase constant, $p_{0}$ is the amplitude of sinusoidal oscillation, $x$ and $r$ are distances in Cartesian and spherical coordinate systems respectively. The wavenumber $k$ and angular frequency $\omega$ are defined as,

$$
k=\frac{2 \pi}{\lambda}
$$

where

$$
\omega=2 \pi f
$$

and $\lambda$ is the wavelength.

\subsubsection{Transmission loss}

Consider a sound source in the sea, the intensity of sound can be measured at any distance d. The transmission loss $T L$ in decibels $(\mathrm{dB})$ is then,

$$
T L=10 \log _{10} I_{0}-10 \log _{10} I
$$

where $I_{0}$ is the reference intensity and it is measured at the distance 1 meter from the source and $I$ is the intensity at the distance $d$ meters from the source. 


\subsubsection{Spreading loss}

In most cases with a point source and an assumption that the medium is infinite and homogeneous, the sound would have a spherical wave front. Under these conditions, the power $P$ generated by the source is radiated equally in all directions and distributed equally over the surface of a sphere surrounding the point source. If there is no loss in the medium, the power $P$ crossing all these spheres is the same. Thus

$$
P=4 \pi r_{1}^{2} I_{1}=4 \pi r_{2}^{2} I_{2}=\ldots 4 \pi r_{n}^{2} I_{n}
$$

where $r_{n}$ is the radius of $n^{t h}$ sphere and $I_{n}$ is the intensity of sound wave at surface of that sphere. If $r_{1}=1$ the changes in power density with the distance $r$ from point source is

$$
T L_{s}=20 \log _{10} r
$$

If the medium is bounded such as in the ocean, the spreading sometimes no longer appears spherical but is better represented as cylindrical spreading. If $H$ is the height between bounds, the power the crossing cylindrical surface range (where here $r$ is in cylindrical coordinates) $r_{1}, r_{2}$ is,

$$
P=2 \pi r_{1} H I_{1}=2 \pi r_{2} H I_{2}=\ldots 2 \pi r_{n} H I_{n}
$$


Thus, the cylindrical spreading loss in $\mathrm{dB}$ is

$$
T L_{c}=10 \log _{10} r
$$

where $T L_{s}$ denotes spherical spreading loss and $T L_{c}$ denotes cylindrical spreading loss. Typically, in the ocean, the wave spreading loss is somewhere between spherical and cylindrical due to losses at the boundary interactions. This is one of the reasons the loss at the seabed boundary is important for understanding downrange TL.

\subsubsection{Absorption loss}

The primary causes of absorption (in the water column) have been attributed to several processes, including viscosity and thermal conductivity. It involves a process of converting the acoustic energy into heat. The absorption loss is represented by a "logarithmic absorption coefficient" $\alpha$ as follows [9],

$$
\alpha=\frac{10 \log _{10} I_{1}-10 \log _{10} I_{2}}{r_{2}-r_{1}}
$$

The quantity $\alpha$ is often expressed in decibels per kiloyard (dB/kyd) or can also be in $\mathrm{dB} / \mathrm{km}$ or $\mathrm{dB} / \mathrm{m}$. 


\subsection{Reflection and scattering}

In a monostatic configuration with the sound source and receiver co-located in the water column, an acoustic transmitted pulse will propagate to the seabed and there it can be both reflected and scattered back to the receiver. A perfect reflection occurs, without scattering, only if the seafloor is perfectly flat which is not generally realistic. Typically, part of the incident pulse will be reflected as a coherent signal and another part will be scattered due to both the roughness at the water-sediment interface and inhomogeneities in the water column and sediment. The bottom backscattering strength is equivalent to the bottom backscattering cross section per unit area per unit solid angle $\theta$, where $\theta$ is the grazing angle. The cross section is assumed to be the sum of contributions from interface roughness and the sediment volume inhomogeneities. The APL-UW model developed by Jackson et al in Ref [4] separates the received envelope into a component due to roughness and a component due to volume scattering. For near vertical incidence, the Kirchhoff approximation is applied to estimate the backscattering due to interface roughness. The model and necessary inputs are described in more detail in the next sections.

\subsubsection{Reflection}

Although the seafloor is never perfectly flat and homogeneous, it is useful to consider the ideal case in that the scattering is neglected. In this ideal case when the acoustic pulse hits the seafloor, part of the energy will be reflected back to the water column and another part is transmitted into the second medium. Assumption is that the incident pressure field is a 
unit amplitude plane wave at angular frequency $\omega$. In the ideal case, if the incident grazing angle is $\theta$, the pressure field can be approximated at interface as,

$$
P_{i}=P_{i 0} e^{i \mathbf{k}_{i} \cdot \mathbf{r}}
$$

where $P_{i 0}$ is the complex pressure amplitude at the origin, $\mathbf{r}$ is the position vector, and $\mathbf{k}_{i}$ is the wave vector giving the direction of propagation of plane wave. As indicated in Fig. 2.3, the $z$ coordinate will be taken perpendicular to the seafloor. For convenience, the coordinate system will be chosen so that the direction of propagation of the plane wave lies in the $x-z$ plane. Then the incident wave vector has $(x, y, z)$ components,

$$
\mathbf{k}_{i}=\frac{\omega}{c_{w}}(\cos \theta, 0,-\sin \theta)
$$

the reflected wave denoted as $P_{r}$ will be a plane wave of the form,

$$
P_{r}=V_{w w}(\theta) P_{i 0} e^{i \mathbf{k}_{r} \cdot \mathbf{r}}
$$

where the reflected wave vector $\mathbf{k}_{r}$ is defined as:

$$
\mathbf{k}_{r}=\frac{\omega}{c_{w}}(\cos \theta, 0, \sin \theta)
$$

for $\theta_{i}=\theta_{s}=\theta$, where $\theta_{i}$ and $\theta_{s}$ are incident and scattering grazing angles respectively. 


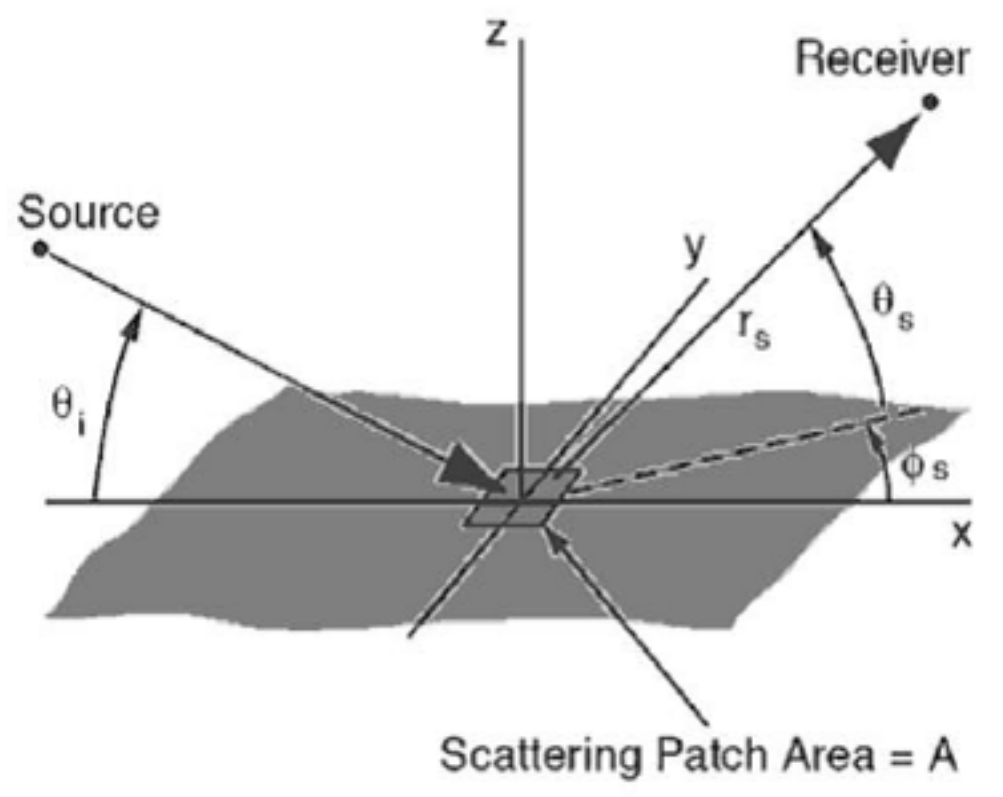

Figure 2.3: Geometry for illustrating angular coordinates used in treating reflections and scattering (adapted from [3]).

The complex parameter $V_{w w}(\theta)$ is the reflection coefficient, with the subscripts $w w$ to indicate that the incident and reflected fields are both measured in the water. The bottom loss $B L$ is defined as:

$$
B L=-20 \log _{10}\left(\left|V_{w w}(\theta)\right|\right) .
$$

In the case of normal incidence, $V_{w w}(\theta=90)$ becomes:

$$
V_{w w}(90)=\frac{\rho_{s} c_{s}-\rho_{w} c_{w}}{\rho_{s} c_{s}+\rho_{w} c_{w}},
$$

where the subscripts $s$ on $\rho$ and $c$ indicate seabed density and sound speed and $w$ indicates 
water parameters.

\subsubsection{Scattering}

Acoustic waves are scattered randomly by irregularities in the seafloor, including the roughness of the water-sediment interface, spatial variation in sediment physical properties and other objects such as bubbles, solid and organic particles, shell pieces, marine life and inhomogeneities in ocean sediment. When the sound wave is scattered, part of the reflected energy is returned to the source as an echo (i.e, is backscattered) some parts are reflected off in another direction and is lost energy. The scattering processes are described in the high level schematic shown in Fig. 2.4. The amount of energy scattered is a function of the size, density, and concentration of foreign bodies present in the sound path, as well as the frequency of the sound wave. The larger the area of the reflector compared to the sound wavelength, the more effective it is as a scatterer. At high frequencies (e.g., above $10 \mathrm{kHz}$ ), all seafloors have substantial irregularities on the scale of acoustic wavelength. The treatment of seafloor scattering at high frequencies typically employ statistical methods to predict the variance of the scattering field. The most commonly used statistical quantity is the scattering cross section, that is proportional to the variance of the scattered field. 


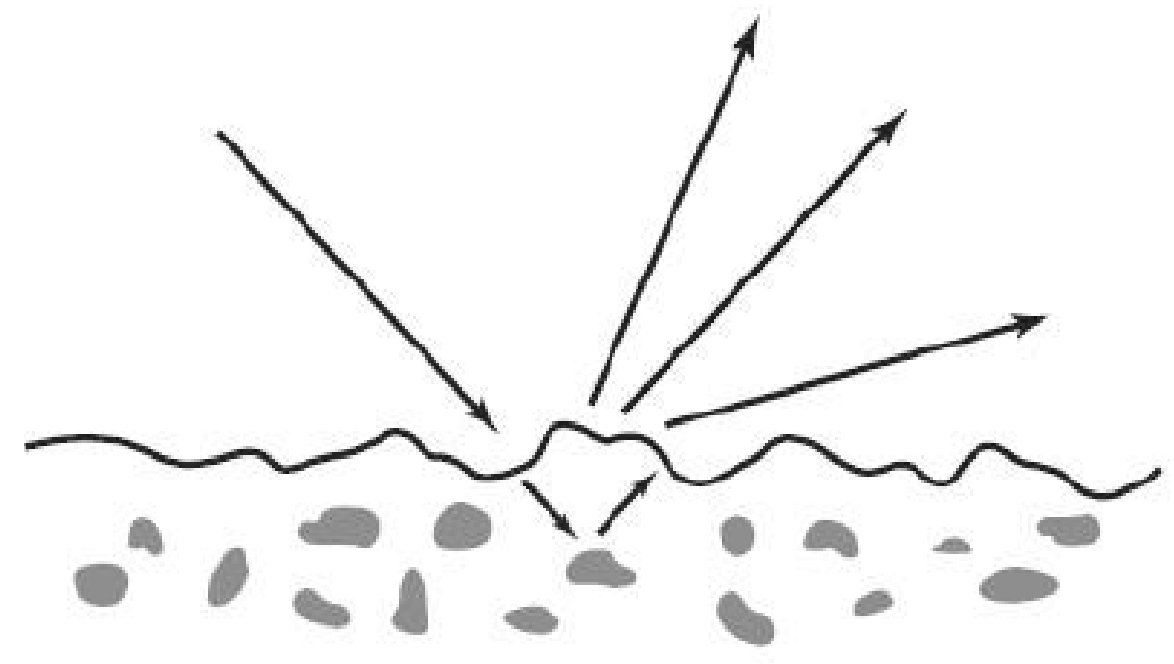

Figure 2.4: High level schematic to describe acoustic scattering due to the roughness of water-sediment interface and heterogeneity of the sediment (adapted from [3]).

Typically, the total pressure field $P$ in the random scattering media is decomposed as follows:

$$
P=<P>+P_{s}
$$

where $\left\langle P>\right.$ is treated as the mean of the complex pressure field and $P_{s}$ is a fluctuation about this mean.

The mean-square fluctuations are equal to the variance of the field and can be expressed as :

$$
<\left|P_{s}\right|^{2}>=<\left|P^{2}\right|>-|<P>|^{2}
$$

The subscript $s$ is attached to fluctuating part of the field as it is the "scattering" part. 
The mean field can be considered as the coherent part. The scattering by the seafloor is usually quantified in term of "scattering strength" whose definition follows from the situation depicted in Fig. 2.4, in which the a small patch with area $A$ is situated in far field of the source. Then, the mean square pressure fluctuation will be proportional to both the area of the patch and the squared incident pressure magnitude $\left|P_{i}\right|^{2}$ and will be inversely proportional to the square of the distance $r$ from the patch. Thus,

$$
<\left|P_{s}\right|^{2}>=\left|P_{i}\right|^{2} A \sigma \frac{1}{r_{s}^{2}} .
$$

Note that $i$ and $s$ subscripts denote "incident" and "scattering". The attenuation and refraction in the seawater are neglected here. The proportional factor $\sigma$ is dimensionless and is sometimes referred to as the "scattering cross section per unit area per unit solid angle" because the integral of $\sigma$ over the upper solid angle hemisphere yields the total mean scattered power $U_{s}$ as follows

$$
U_{s}=\frac{A\left|P_{i}\right|^{2}}{2 \rho_{w} c_{w}} \int_{2 \pi} \sigma\left(\theta_{i}, \theta_{s}, \phi_{s}\right) d \Omega_{s}
$$

where,

- $\rho_{w}$ is the density of seawater

- $\rho_{w} c_{w}$ is the acoustic impedance of seawater

- $\theta_{i}$ is the incident grazing angle 
- $\theta_{s}$ is the scattered grazing angle

- $\phi_{s}$ is the bistatic angle

The factor 2 in the denominator of eq. (2.25) appears because time averages of squared sinusoidally oscillating functions are one-half the square of the peak magnitude. The quantity $\sigma\left(\theta_{i}, \theta_{s}, \phi_{s}\right)$ is simply referred as the backscattering cross section [3].

It is important to remember that the scattering cross section is defined here as a statistical average. Referring to Fig. 2.3, the scattering cross section depends on three angular variables: a grazing angle, for the incident field $\theta_{i}$, and grazing and azimuthal angles of scattering fields $\theta_{s}$ and $\phi_{s}$. These dependencies can be shown explicitly by writing the scattering cross section as $\sigma\left(\theta_{i}, \theta_{s}, \phi_{s}\right)$. The angle $\phi_{s}$ is often referred to as the "bistatic angle". If the seafloor has a preferred direction an azimuthal angle $\phi_{i}$ is also required for the incident field. In either case, $\sigma$ is referred to as the "bistatic" scattering cross section. A simpler and more common case is backscattering, or the "monostatic" case, in which the transmitter and receiver are situated at the same point in space. For that case, $\theta_{s}=\theta_{i}=$ and $\phi_{i}=\phi_{s}+\pi=\phi$ and $S_{b}$ is referred as a "backscattering strength", and is expressed in $\mathrm{dB}$

$$
S_{b}=10 \log _{10} \sigma
$$

where only two angular variables, $\theta$ and $\phi$ are needed. If the seafloor has no preferred direction then the variable $\phi$ can be eliminated.

The cross section is assumed to be the sum of the interface and sediment inhomogene- 
ity scattering. In the literature ([4], [9] [11], [12]), several expressions of scattering cross section $\sigma$ are described. In this study, the following expression from [4] and [12] will be used:

$$
\sigma=\left(\sigma_{s u r}+\sigma_{v o l}\right)
$$

where $\sigma_{\text {sur }}$ refers to the scattering strength due to water-seabed interface and, and $\sigma_{v o l}$ is volume scattering strength due to sediment inhomogeneities,

$$
S_{b}=10 \log _{10}\left(\sigma_{\text {sur }}+\sigma_{v o l}\right) .
$$




\section{Chapter 3}

\section{Underwater acoustic systems and empirical approach for seabed classification}

This chapters provides a short review of acoustic systems and a phenomenological approach using single beam echosounders for seabed classification.

\subsection{Acoustic instrument}

Acoustic instruments that are commonly used for seabed classification are grouped into four categories as described by Anderson et al in [13] and Hammilton et al in [6]. They are briefly described in the following subsections.

\subsubsection{Single-beam echosounder (SBES)}

Single beam echosounders operate one or more transducers which are designed with a narrow beam at specific frequencies. SBES are reported as the least expensive and least complex underwater acoustic instrument. They are the most common instrumentation employed due to low cost, simple operation, and less complexity in data processing [14]. 


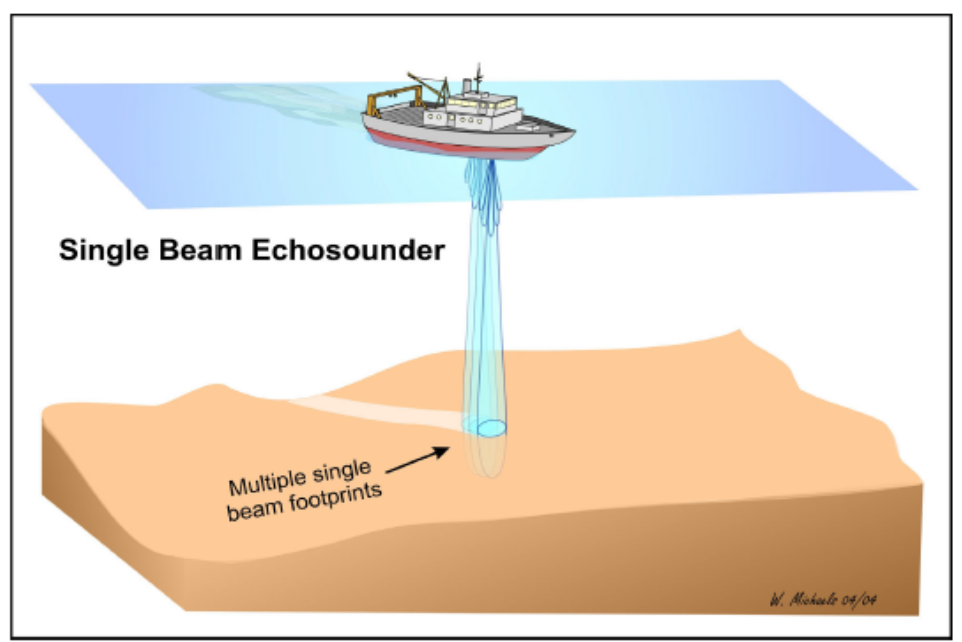

Figure 3.1: An illustration of SBES . The figure is adapted from [6].

\subsubsection{Sidescan sonar (SSS)}

A simple sidescan sonar is equipped with single-beam echosounder on each side of a vehicle (or towfish), and the transducers are tilted towards the seabed. Compared to the SBES, the swath footprint provides more seabed coverage and it is relatively easy to operate.

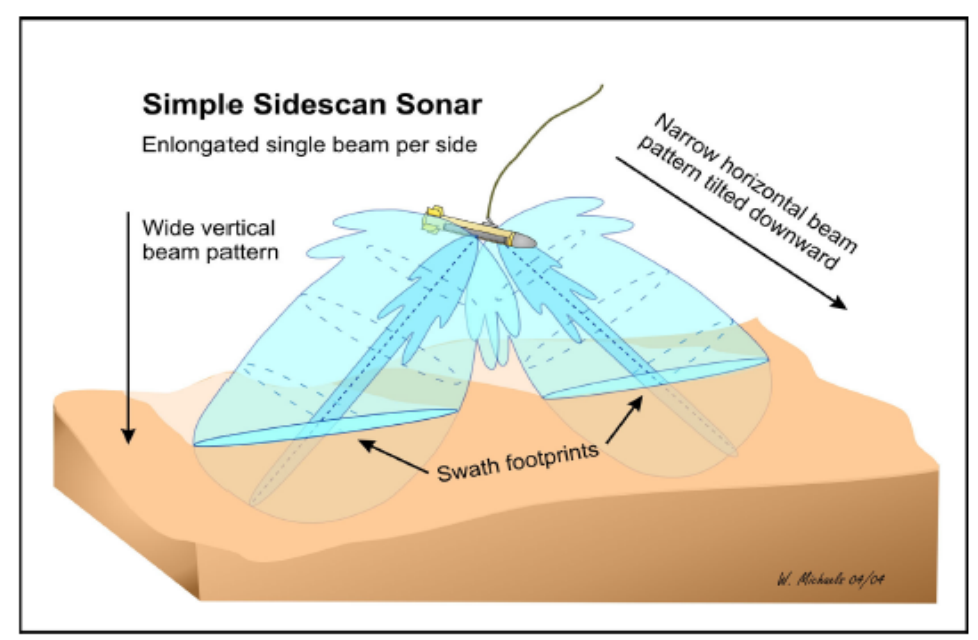

Figure 3.2: Simple sidescan consists of single beam echosounder per side . The figure is adapted from [6]. 


\subsubsection{Multi-row SSS}

More advanced SSS systems consist of multiple elements arranged in a row to improve the accuracy in estimating the incidence angle, horizontal range and bathymetric measurements.

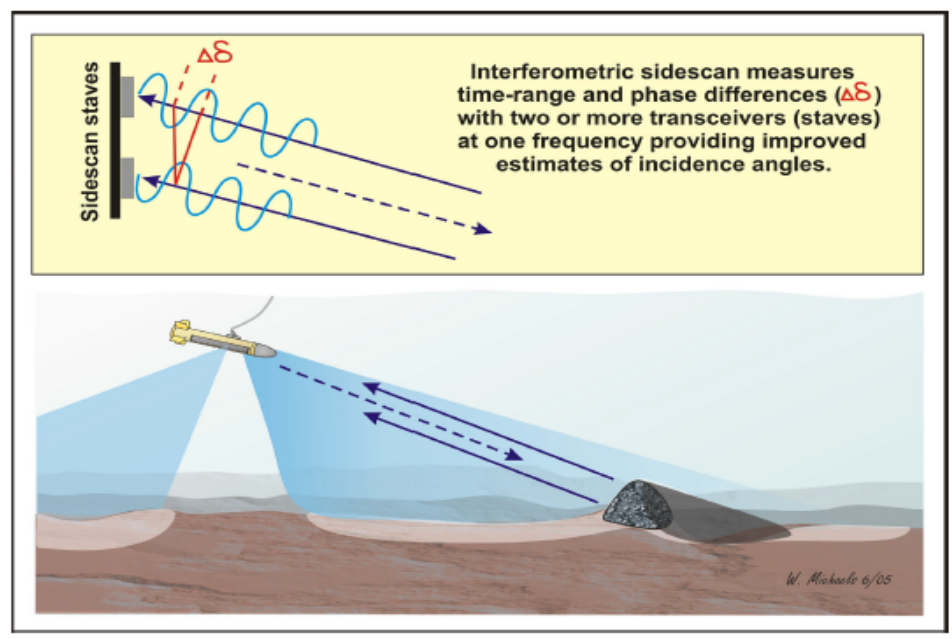

Figure 3.3: Multiple row sidescan consists of multiple single beam echosounder per side. The figure is adapted from [6].

\subsubsection{Multibeam sonar (MBES)}

Multibeam sonars are designed for collecting the bathymetric and backscatter for hydrographic seabed mapping and classification. MBES systems are more complex and expensive compared to SBES however they have more advantages such as higher resolution, ability to detect the angle of incidence and detection of multiple scattering from two different targets. 


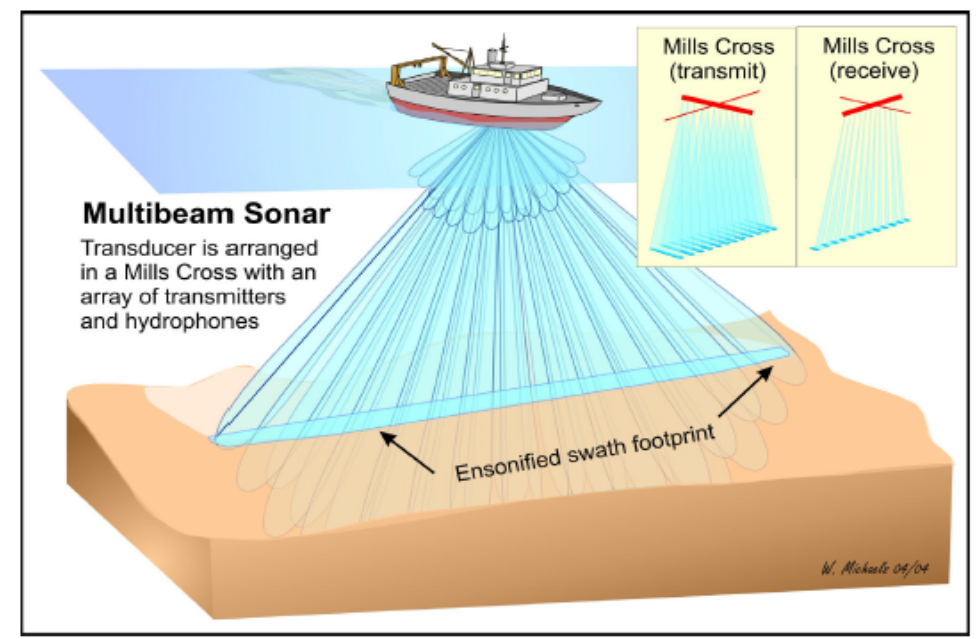

Figure 3.4: The multibeam sonar head is arranged in a Mills Cross with an array of transmitters and array of beam steered hydrophones. This figure is adapted from [6].

\subsection{Phenomenological approach using SBES for seabed classification}

As mentioned earlier, acoustic remote sensing classification methods are numerous but can fall under two general categories: phenomenological approaches and physics based approaches [1], [2]. While the model-based method uses an inversion procedures to characterize the seafloor, the empirical method relies on the the study of signal features that are correlated with sediment type. The empirical method requires establishing a data base "ground truth". The typical features of returned signal used for this approach are time spread, echo energy, and skewness [11], [15]. This approach has been used by several commercial acoustic bottom classification system, RoxAnn and QTC-View are two of them. The review of these two system was made by Hamiton et al in [6].

\subsubsection{The multi-echo energy approach of RoxAnn system}

The RoxAnn system uses a multi-echo energy classification method. RoxAnn functions by integrating components of the first and second seabed echoes to derive two parameters of the seabed substrate E1 and E2. E1 is an integration of the tail of the first seabed echo, and is taken to represent seabed "roughness". E2 is an integration of the whole of the second bottom echo and provides an index of seabed "hardness" [7]. 


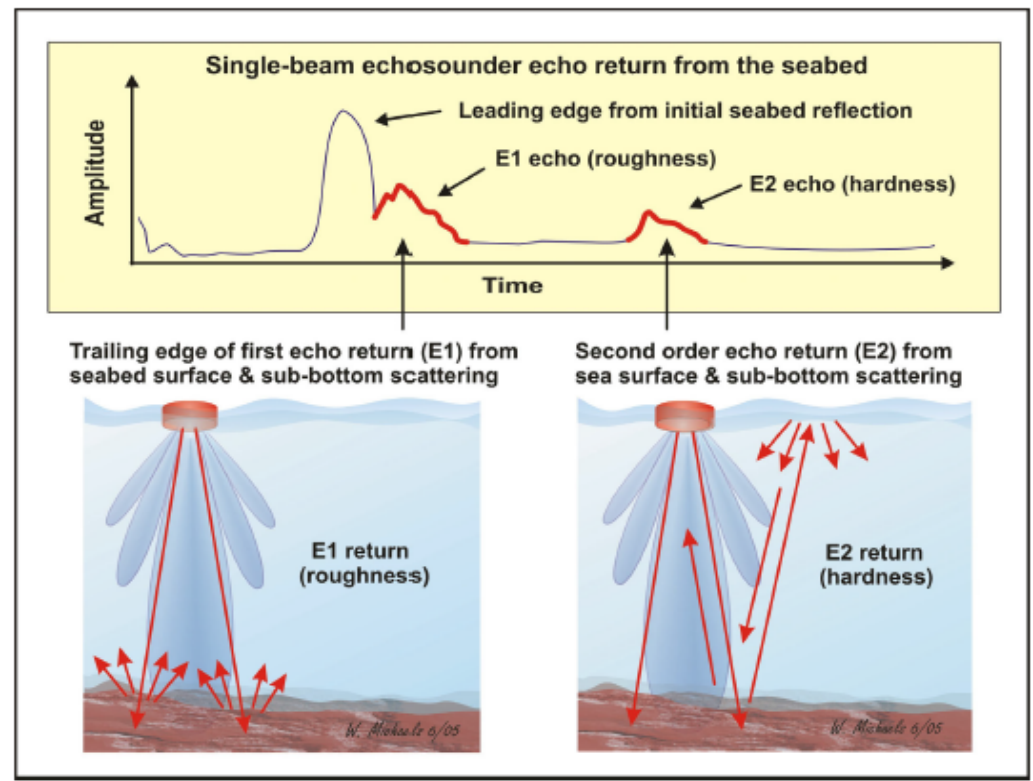

Figure 3.5: Illustration of working principle of RoxAnn system. This figure is adapted from [6].

The first echo is reflected from bottom while the second echo bounced twice, first it interacted with sea surface then the sea bottom. The data then is presented as a scatter plot E1 vs. E2. Based on the the cluster pattern of scatter plots, the operator applies the "box set" to these data in such the way that each box has a maximum and minimum E1 and E2. Each box represents the particular sediment type which is defined based on the ground-truth samples. This approach is purely empirical and works well for flatter bottoms, however for the rougher bottoms, E2 appears an unreliable classifier due to energy lost resulted from the scattering processes [16]. 


\subsubsection{Quester Tangent Corporation (QTC) approach}

In contrast to RoxAnn, the QTC system examines the shape characteristics of only the first returning signal from an echosounder transducer. QTC normalizes the first echo to unity peak amplitude before calculating shape parameters [6]. A set of 166 parameters are extracted from acoustic data. Post-processing analysis of the acoustic data is carried out using the software packages CAPS and QTC IMPACT. Most of the 166 parameters carry limited information or redundant information. Principal component analysis (PCA) is used to determine the best combination of the 166 features for discrimination of the echoes. The 166 feature combinations are therefore automatically reduced to three composite values, known as Q1, Q2, and Q3 . The Q-values are chosen automatically by principal component analysis by the QTC software from five algorithms as Histogram, Quantiles, Integrated Energy Slope, and Walet packages [17]. For more details about the principal component analysis for seabed classification using QTC system see [15]. The QTC system provides new insights but the usefulness of the acoustic classification depends upon the amount and quality of ground-truth data [18]. The user relates acoustic class to the physical properties of the seabed through a calibration process. The ground truth data can be obtained using bottom grabs or video recordings. The system works well with flatter bottoms.

In a recent study, Hamilton et al in [16] compared the RoxAnn and QTC acoustic bottom classification systems in terms of performance. They found that the QTC classes generally had consistent sediment grain size properties and gave a better classification than the RoxAnn system. 


\section{Chapter 4}

\section{Theoretical model and methodology for model-based seabed classification}

This chapter describes the theoretical model and model-based approach for sediment classification.

\subsection{Theoretical model}

\subsubsection{Model Inputs: Sea floor parameters}

The geoacoustic parameters serving as the model inputs are listed in Table. 4.1. They are needed to represent the seafloor type and are largely taken from the APL-UW Environmental Handbook [4].

\begin{tabular}{clll}
\hline Symbol & Description & Short name \\
\hline$\rho$ & $\begin{array}{l}\text { Ratio of sediment mass density to water density } \\
\text { Ratio of sediment sound speed to water sound }\end{array}$ & $\begin{array}{l}\text { Density ratio } \\
\text { Sound speed ratio }\end{array}$ \\
$\delta$ & $\begin{array}{l}\text { Speed } \\
\text { Ratio of imaginary wavenumber to real } \\
\text { wavenumber for sediment }\end{array}$ & Loss parameter \\
$\sigma_{2}$ & $\begin{array}{l}\text { Ratio of sediment volume scattering cross sec- } \\
\text { tion to sediment attenuation coefficient }\end{array}$ & Volume parameter \\
$\gamma$ & $\begin{array}{l}\text { Exponent of bottom relief spectrum } \\
W_{2}\end{array}$ & $\begin{array}{l}\text { Strength of bottom relief spectrum } \mathrm{cm}^{4} \text { at } \\
\text { wavenumber } k=\frac{2 \pi}{\lambda}\left(\mathrm{cm}^{-1}\right)\end{array}$ & $\begin{array}{l}\text { Spectral parameter } \\
\text { Spectral strength }\end{array}$ \\
$M_{z}$ & Mean grain size (in units of $\phi)$ & \\
\hline
\end{tabular}

Table 4.1: Parameterization of inputs in term of bulk mean grain size $M_{z}$ (defined in [4]). 
The mean grain size is often expressed in units of $\phi$ as

$$
M_{z}=-\log _{2} \frac{d}{d_{0}}
$$

where $d_{0}$ is reference length equal to $1 \mathrm{~mm}, d$ is mean grain size (diameter) in $\mathrm{mm}$. The mean grain size takes the values from $-1 \phi$ to $9 \phi$.

According Jackson et al in [3], for near- vertical backscattering the mean square received envelope is taken as a sum of components due to roughness and volume scattering,

$$
<\left|V_{r}(t)\right|^{2}>=<\left|V_{r r}(t)\right|^{2}>+<\left|V_{r v}(t)\right|^{2}>
$$

where, $<\left|V_{r}(t)\right|^{2}>$ is the total received envelope, $<\left|V_{r r}(t)\right|^{2}>$ is the received envelope contribution due to interface roughness, $<\left|V_{r v}(t)\right|^{2}>$ is the received envelope contribution due to volume scattering.

\subsubsection{Model for near vertical backscattering due to roughness}

For near vertical backscattering due to a rough interface, the Kirchhoff approximation is applied. Appendix. $\mathrm{B}$ describes in details of this approximation method. This section presents the very final steps where the model is derived by Jackson et al in [3]. 


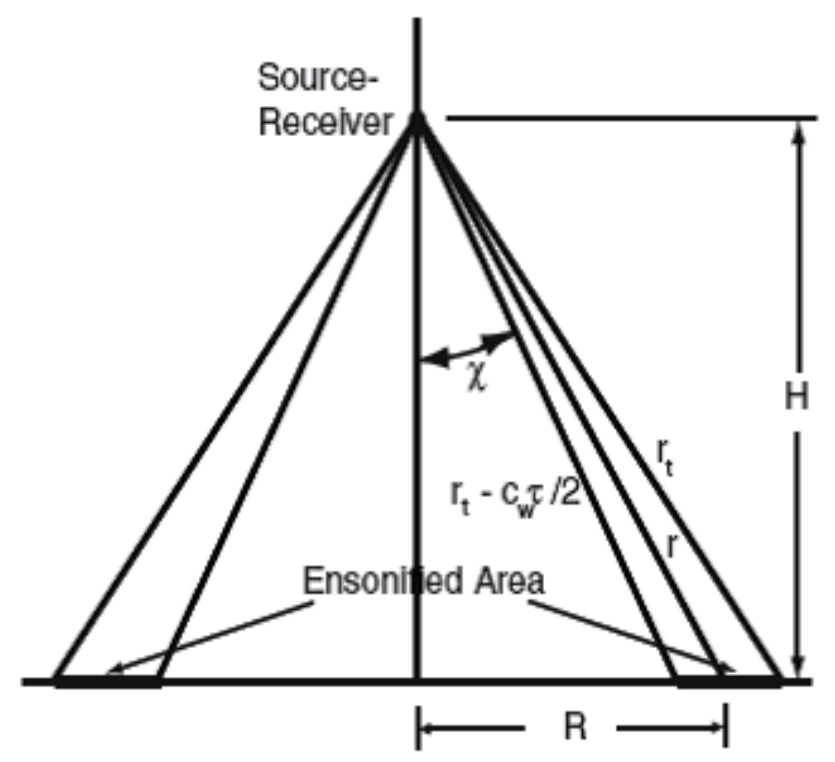

Figure 4.1: Illustration of interface scattering geometry.

Figure 4.1 illustrates the geometry of the interface scattering components of the model. For simplicity, it is assumed that the transmitted signal has a rectangular envelope of length $\tau$ and the source-receiver is at the origin for convenience. With these assumptions, the backscattered signal received at time $t$ (measured from the beginning of the transmitted pulse) is due to scatters lying within the slant range interval,

$$
r_{t}-\frac{c_{w} \tau}{2}<r<r_{t}
$$

where,

$$
r_{t}=\frac{c_{w} t}{2}
$$

is range associated with the time of interest $t$. The interface scattering eq. (B.18) can be 
rewritten as,

$$
<\left|V_{r r}(t)\right|^{2}>=\frac{2\left(s_{0} s_{r}\right)^{2} e^{-4 k_{w}^{\prime \prime} H}}{H^{4}} \int \sigma\left|b_{r}(\theta, \phi) b_{x}(\theta, \phi)\right|^{2} d^{2} R
$$

where $H$ is the height of the source-receiver above the seafloor, and it is assumed that the scattering is confined to narrow region immediately below the source-receiver.

$$
\begin{array}{ll}
s_{0} & : \text { RMS source pressure } \\
s_{r} & : \text { Receiving sensitivity as voltage/pressure ratio } \\
b_{r} & : \text { Receiving complex directivity function } \\
b_{x} & : \text { Source complex directivity function } \\
k_{w}^{\prime \prime} & : \text { Grazing angle } \\
\theta & \\
\phi_{w w} & : \text { Azimuthal angle } \\
V_{w}\left(90^{0}\right) & : \text { Reflection coefficient of flat interface at near nadir }
\end{array}
$$

The source level $\left(20 \log _{10} s_{0}\right)$ is measured in the vertical direction which results in,

$$
\left|b_{r}\left(\frac{\pi}{2}, 0\right) b_{x}\left(\frac{\pi}{2}, 0\right)\right|=1
$$

It will be assumed that the scattering is isotropic so that scattering strength $\sigma$ does not depend on the azimuthal angle $\phi$. The integral over $\phi$ only involves the directivity so the 
azimuthal averaged directivity for small angle of incidence near vertical is:

$$
b(\theta)=\frac{1}{2 \pi} \int_{-\pi}^{-\pi}\left|b_{r}(\theta, \phi) b_{x}(\theta, \phi)\right|^{2} d \phi .
$$

This will be approximated by a Gaussian function,

$$
b(\theta)=e^{-\frac{(\pi / 2-\theta)^{2}}{2 \sigma_{b}^{2}}},
$$

where $\sigma_{b}$ is beamwidth parameter.

A similar approximation will be used for the scattering cross section. The high frequency Kirchhoff approximation eq. (B.17) near the nadir can be written as

$$
\sigma_{\text {sur }}=\frac{\left|V_{w w}\left(90^{0}\right)\right|^{2}}{8 \pi \sigma_{s}^{2}} e^{-\frac{(\pi / 2-\theta)^{2}}{2 \sigma_{s}^{2}}}
$$

This should be regarded as a parameterization of the scattering cross section near vertical incidence. In this approximation, the parameter $\sigma_{s}$ should be considered as a measure of the angular width of the backscattering cross section peak at vertical incidence rather than as an RMS slope of the interface. With these approximations, the two dimensional integral in eq. 4.5) can be simplified to one dimensional integral as,

$$
<\left|V_{r r(t)}\right|^{2}>=\frac{\left(s_{0} s_{r}\right)^{2} e^{-4 k_{w}^{\prime \prime} H}\left|V_{w w}\left(90^{0}\right)\right|^{2}}{2 H^{4} \sigma_{s}^{2}} \int_{R_{1}}^{R_{2}} e^{-\frac{(\pi / 2-\theta)^{2}}{2 \sigma_{s b}^{2}}} R d R
$$

where

$$
\frac{1}{\sigma_{s b}^{2}}=\frac{1}{\sigma_{s}^{2}}+\frac{1}{\sigma_{b}^{2}}
$$

The parameter $\sigma_{b}^{2}$ is understood to be the variance of the receiver/ source directivity, $\sigma_{s}$ is variance of scattering cross section, and $\sigma_{s b}^{2}$ is interpreted as a combined variance of 
two Gaussian distributions that described by equations 4.9 and 4.8 . $R$ is the cylindrical radial coordinate defined in Fig. 4.1 with the limits defined by eq. (4.3). Defining the angle of incidence measured from vertical as $\chi=\pi / 2-\theta$, one can find the relationship between the angular limits and elapsed time and pulse length.

The elapsed time between the beginning of the transmission and the leading edge of the seafloor return is

$$
t_{0}=\frac{2 H}{c_{w}}
$$

where $c_{w}$ is seawater sound speed.

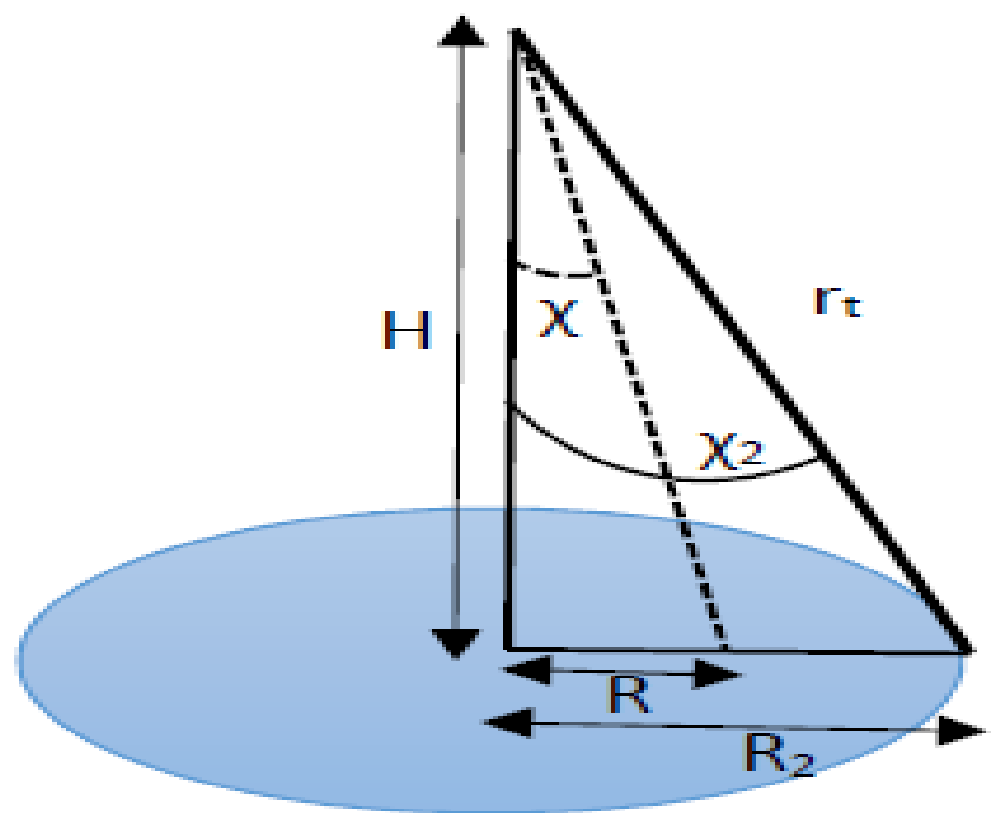

Figure 4.2: An example of signal footprint for $t_{0}<t<t_{0}+\tau$.

Before sufficient time has elapsed to allow the return from the seafloor, $t<t_{0},<$ 
$\left|V_{r r}\right|>=0$. For the time period within one pulse length $\tau$, i.e. $t_{0}<t<t_{0}+\tau$, for a small angle at near normal incidence (where $\chi_{1}=0$ ), the ensonified area is a circle [3] as shown in Fig. 4.2, and an approximation for the small angle $\chi_{2}<<1$,

$$
\chi_{2}^{2} \approx \frac{R_{2}^{2}}{H^{2}}=\frac{r_{t}^{2}-H^{2}}{H^{2}}
$$

or,

$$
\begin{gathered}
\chi_{2}^{2}=\frac{\left(c_{w} t\right)^{2} / 4-\left(c_{w} t_{0}\right)^{2} / 4}{\left(c_{w} t_{0}\right)^{2} / 4} \\
\chi_{2}^{2}=\frac{t^{2}-t_{0}^{2}}{t_{0}^{2}}=\frac{\left(t-t_{0}\right)\left(t+t_{0}\right)}{t_{0}^{2}} .
\end{gathered}
$$

For the near vertical backscattering $t+t_{0} \cong 2 t_{0}$, the approximation for $\chi_{2}^{2}$ becomes,

$$
\chi_{2}^{2}=\frac{2\left(t-t_{0}\right) t_{0}}{t_{0}^{2}}=2\left(t / t_{0}-1\right) .
$$

For longer time $t>t_{0}+\tau$, the ensonified area is an annulus defined by the angular limits as follows (the similar approach for approximation of $\chi_{1}$ is applied),

$$
\chi_{1}^{2}=2\left[(t-\tau) / t_{0}-1\right]
$$

and

$$
\chi_{2}^{2}=2\left(t / t_{0}-1\right)
$$

If the integration variable in eq. 4.10 is changed to $\chi^{2}$, and the limits of integration are changed from $R_{1}, R_{2}$ to $\chi_{1}^{2}, \chi_{2}^{2}$ respectively, the integrand becomes a simple exponen- 
tial, from which one can obtain,

$$
<\left|V_{r r}(t)\right|^{2}>=V_{1}^{2}\left|V_{w w}\left(90^{0}\right)\right|^{2} \frac{g\left(t-t_{0}, T_{s b}, \tau\right)}{1+\frac{\sigma_{s}^{2}}{\sigma_{b}^{2}}}
$$

where,

$$
\begin{gathered}
g\left(t-t_{0}, T, \tau\right)=0 \quad \text { for } t<t_{0} \\
g\left(t-t_{0}, T, \tau\right)=1-e^{-\left(t-t_{0}\right) / T} \quad \text { for } t_{0}<t \leq \tau+t_{0} \\
g\left(t-t_{0}, T, \tau\right)=e^{-\left(t-t_{0}-\tau\right) / T}-e^{-\left(t-t_{0}\right) / T} \quad \text { for } t>\tau+t_{0} \\
T_{s b}=\sigma_{s b}^{2} t_{0},
\end{gathered}
$$

and

$$
V_{1}^{2}=\frac{\left(s_{0} s_{r}\right)^{2} e^{-4 k_{w}^{\prime \prime} H}}{2 H^{2}}
$$

is a normalization factor equal to the mean square voltage that would be measured if the interface was perfectly flat with reflection coefficient having unity magnitude. The normalized intensity $\left(<\left|V_{r r}(t)\right|^{2}>\right)$ at first approaches an asymptote exponentially and then after a time of one pulse length decays towards zero. The rise time of the $<\left|V_{r r}(t)\right|^{2}>$ provides a measure of angular width $\sigma_{s}$ of the backscattering cross section. Narrow width gives the rapid rise and vice versa.

\subsubsection{Volume scattering model}

Appendix. C describes in details the approximation for scattering due to sediment inhomogeneities, this section describes only last steps leading to the final expression needed to implement the model. 


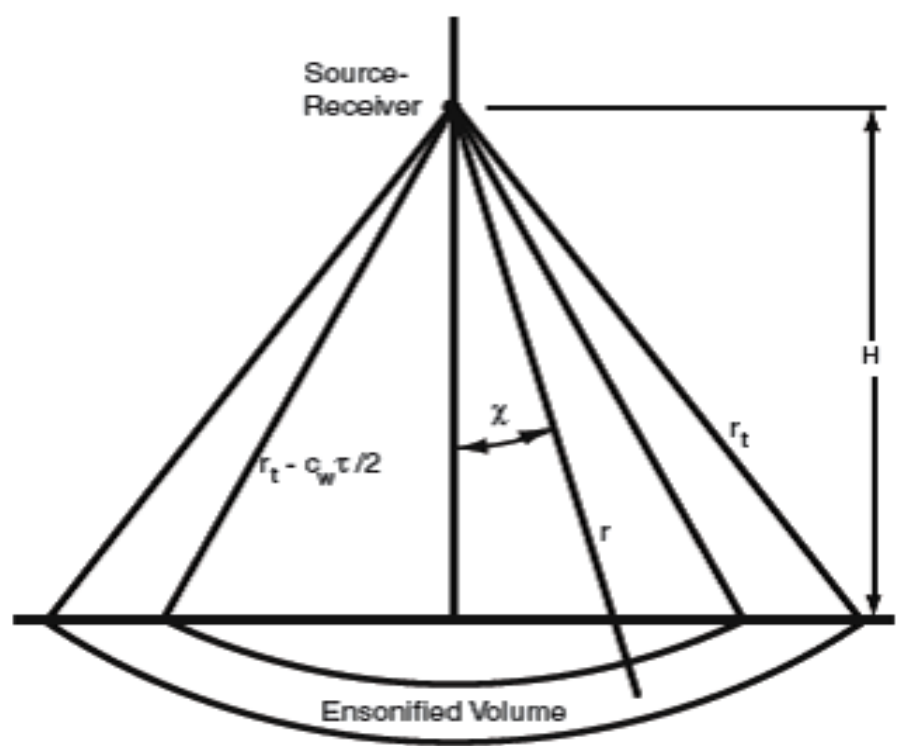

Figure 4.3: Illustration of volume scattering geometry

The volume scattering cross section is parameterized as following,

$$
\sigma_{v o l}=\frac{\left|V_{w p}\left(\theta_{i}\right)\right|^{2}\left|V_{w p}\left(\theta_{s}\right)\right|^{2} \sigma_{v}}{2 k_{w}|\rho|^{2} \operatorname{Im}\left[P\left(\theta_{i}\right)+P\left(\theta_{s}\right)\right]}
$$

where

$$
V_{w p}\left(90^{0}\right)=1+V_{w w}\left(90^{0}\right)
$$

and $\sigma_{v}$ is the volume scattering cross section and is treated as an empirical quantity that must be obtained by fitting data [19]. The following dimensionless parameter $\sigma_{2}$ is used to quantify the sediment volume scattering,

$$
\sigma_{2}=\frac{\sigma_{v}}{\alpha_{p}}
$$

where $\alpha_{p}$ is the attenuation in $\mathrm{dB} \mathrm{m}^{-1}$.

The squared averaged received signal due to volume scattering can be found from 
following,

$$
<\left|V_{r v}\right|>^{2}=\frac{8 \pi \sigma_{v} V_{1}^{2}\left|V_{w p}\left(90^{0}\right)\right|^{4}}{\rho^{2}} \int_{r_{1}}^{r_{2}} \int_{0}^{\chi_{2}} b(\theta) e^{-4 k_{p}^{\prime \prime}(r-H / \cos \chi)} \sin \chi d \chi d r .
$$

The factor $\left|V_{w p}\right|^{4} / \rho^{2}$ accounts for round trip transmission through the interface. The exponential factor accounts for attenuation in the sediment with $k^{\prime \prime}$ being the coefficient of the imaginary part of the sediment wavenumber. The angular limits are,

$$
\chi_{2}=\cos ^{-1}(H / r)
$$

For $t_{0}<t \leq t_{0}+\tau$, the ensonified volume is a spherical section

$$
r_{1}=H
$$

and

$$
r_{2}=r_{t}
$$

For $t>t_{0}+\tau$, the ensonified volume is a spherical shell

$$
r_{1}=r_{t}-c_{w} \tau / 2
$$

and

$$
r_{2}=r_{t}
$$

Making the small angle approximation $(\chi<<1)$, the integrand can be written in term of a simple exponential in $\chi^{2}$ and $r$ which results in,

$$
<\left|V_{r v}\right|>^{2}=\frac{8 \pi \sigma_{v} V_{1}^{2}\left|V_{w p}\left(90^{0}\right)\right|^{4}}{\rho\left(\sigma_{p}^{-2}+\sigma_{b}^{-2}\right)}\left[\sigma_{b}^{2} g\left(t-t_{0}, T_{b}, \tau\right)-\sigma_{p}^{2} g\left(t-t_{0}, T_{p}, \tau\right)\right]
$$


with

$$
T_{b}=\sigma_{b}^{2} t_{0}
$$

and

$$
\begin{gathered}
T_{p}=\sigma_{p}^{2} t_{0} \\
\sigma_{p}^{2}=\frac{1}{4 k_{p}^{\prime \prime} H},
\end{gathered}
$$

where $k_{p}^{\prime \prime}$ is the imaginary part of seabed compressional wave-number.

\subsection{Optimization methodology}

All parameters are searched for in the optimization process accept spectral parameter $\gamma$ which takes the value of 3.25 if a measurement is not available [3] as is the case here.

\subsubsection{Model function}

The theoretical model of the bottom return is mathematically represented by $<\left|V_{r}(t)\right|>^{2}$ in equation (4.2), and its components are described by (4.19) and (4.31). The theoretical model has five unknown parameters that represent the seabed characteristics. The optimization process then seeds the best combination of the unknown model parameters so that the model will have the best fit to the bottom echo captured from measured data. For verification of the correctness of the model described in this research, a plot of modeled signal with the same set of parameters as specified in Fig.G.7 page 506 of [3] was made as shown in Fig. 4.4a. Then, the parameters were varied to see the change in shape of the

modeled signal. The panels 4.4b, 4.4c, 4.4d in Fig. 4.4 are to illustrate how the scattering 
cross section and volume parameters effect to the signal amplitude and shape. The main pulse (first part of the signal) represents the coherent part of signal. The normal incident reflection coefficient value is shown as black dashed line in the plots and indicates the level of an ideally reflected signal for a perfectly flat interface. It can seen that, if the interface is perfectly flat and there is no scattering due to roughness or inhomogeneities, the level of total modeled return signal is the coherent reflection as shown in Fig. 4.4b. If the interface is rough, some energy is scattered away and the return signal level is lower than the ideal reflected signal. The shape of signal also changes.

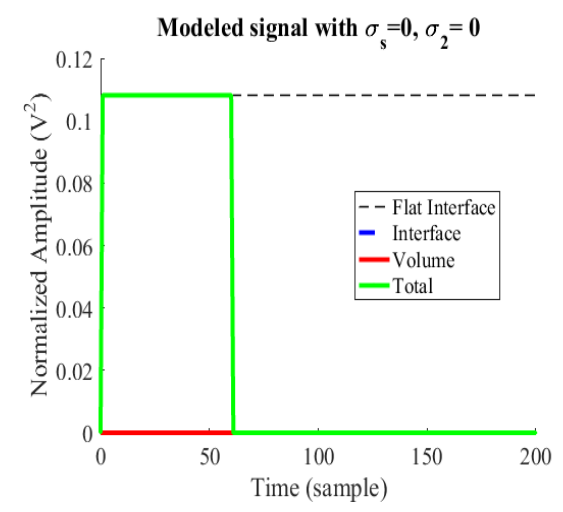

(a)

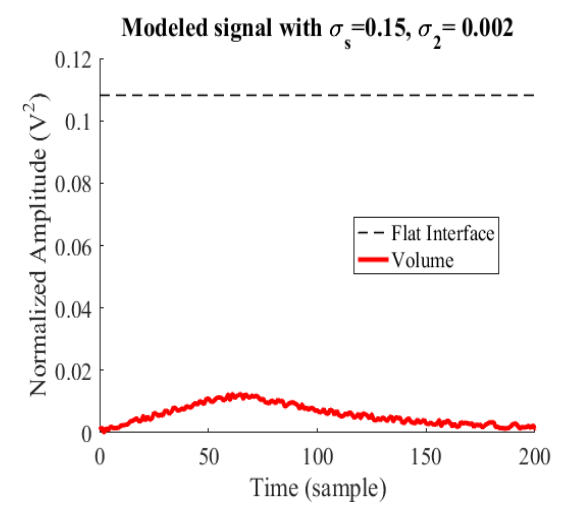

(c)

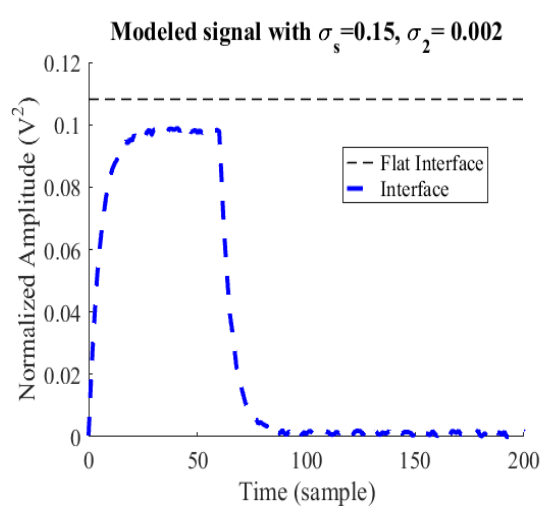

(b)

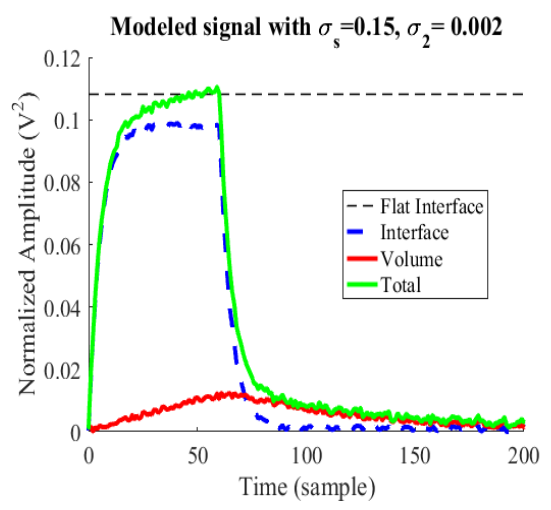

(d)

Figure 4.4: Modeled signal with rectangular pulse. 
Figure 4.4 is the plot of a rectangular pulse with acoustic frequency of $20 \mathrm{kHz}$, and pulse length of $3 \mathrm{~ms}$. The source height is $H=10 \mathrm{~m}$, sound speed in water $c_{w}=1540 \mathrm{~m}$ and the beamwidth parmeter is $\sigma_{b}=0.445$. The other parameters used in the simulation are , sediment water density ratio $\rho=1.451$, sound speed ratio $v=1.1073$, and loss parameter $\delta=0.016$. Panel $4.4 \mathrm{a}$ is the modeled signal returning from flat interface, there is neither interface nor volume scattering. Other panels show the case when there are interface and volume scattering, panel $4.4 \mathrm{~b}$ is plot of interface component, panel $4.4 \mathrm{c}$ is plot of volume component, and panel $4.4 \mathrm{~d}$ is the total modeled signal. The volume parameter $\sigma_{2}=0.002$ and cross section parameter $\sigma_{s}=0.15$ were used for simulation.

The Figure 4.5 are examples of modeled signals with different interface and volume scattering parameters.

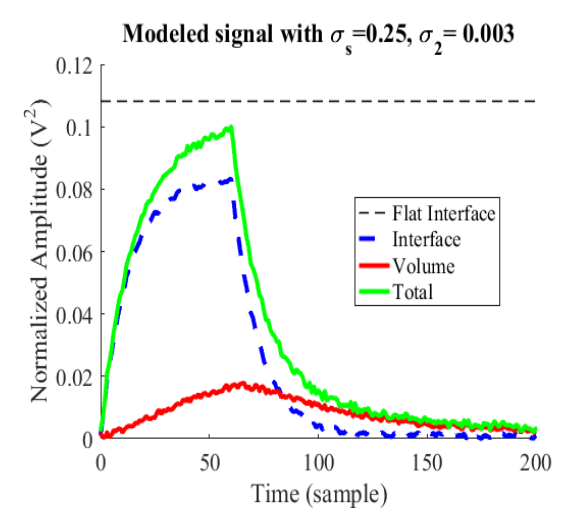

(a)

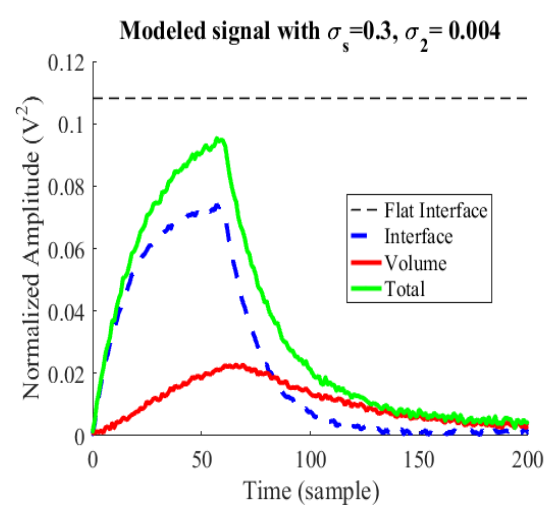

(b)

Figure 4.5: Modeled signal different interface and volume scattering parameters.

In practice, the envelope of the acoustic pulse is not always rectangular and often can have a Gaussian shape. In this case the Gaussian window can be applied to the model as shown in Fig. 4.6 . 


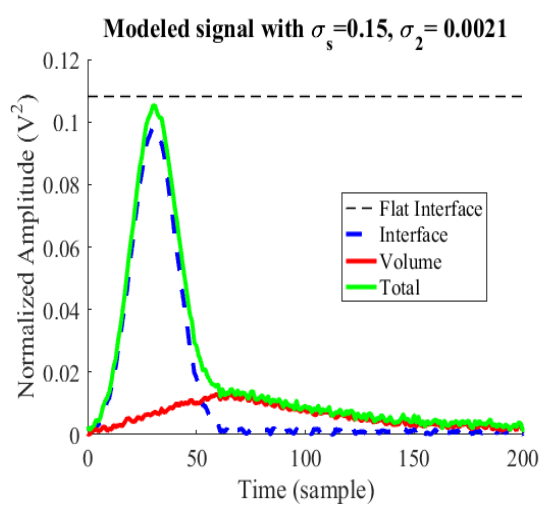

(a)

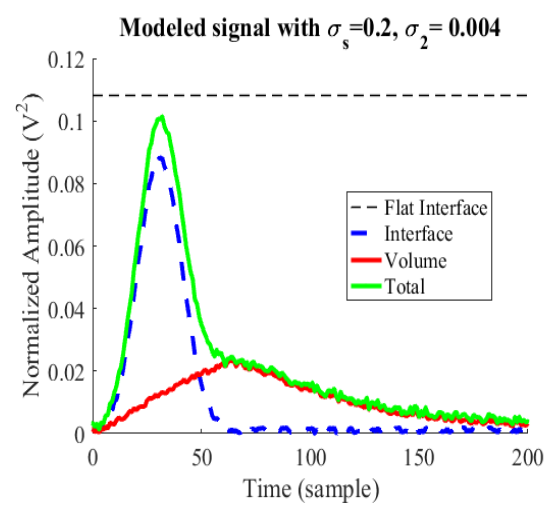

(b)

Figure 4.6: Modeled signal with Gaussian window.

\subsubsection{Cost function}

A cost function is needed to determine the degree that the model fits the data. There are two cost functions used here for two different steps in the optimization process as follows:

\section{Step 1 cost function}

This cost function $E_{1}$ is for searching for the optimal mean grain size $M_{z}$ (which can also be related to the sediment type) and is based on a comparison of the normal incident reflection coefficient. The $E_{1}$ cost function is defined,

$$
E_{1}=\left[V_{\text {mod }}\left(90^{0}\right)-V_{\text {mea }}\left(90^{0}\right)\right]^{2}
$$

where $V_{\text {mea }}\left(90^{\circ}\right)$ is reflection coefficient obtained from echo energy of measured data and $V_{\text {mod }}\left(90^{\circ}\right)$ is the model of Rayleigh reflection coefficient.

Based on the relation between mean grain size, acoustic sediment impedance, and 
bottom reflection coefficient as decribed in [8], [20], $V_{\text {mod }}\left(90^{\circ}\right)$ can be derived as follows,

$$
V_{\text {mod }}\left(90^{0}\right)=\frac{\rho\left(M_{z}\right) v\left(M_{z}\right)-1}{\rho\left(M_{z}\right) v\left(M_{z}\right)+1}
$$

where sediment -water density ratio $\rho$ and sound speed ratio $v$ are known as

$$
\rho=\frac{\rho_{s}}{\rho_{w}}
$$

and

$$
v=\frac{c_{s}}{c_{w}}
$$

where $c_{w}$ is sound speed in water, $\rho_{w}$ is water density. The value of seawater sound speed and density can be found from literature (or easily measured). Note that for the simulation in Fig. 4.4, $c_{w}$ takes the value of $1540 \mathrm{~m} / \mathrm{s}$ and sediment sound speed ratio $v$ and density ratio $\rho$ can be parameterized through different grain sizes according to [4],

$$
\begin{aligned}
& \rho=0.007797 M_{z}^{2}-0.17057 M_{z}+2.3139 \text { for }-1<=M_{z}<=1.0 \text {, } \\
& \rho=-0.165406 M_{z}^{3}+0.2290201 M_{z}^{2}-1.1069031 M_{z} \\
& +3.0455 \text { for } 1<=M_{z}<=5.3 \text {, } \\
& \rho=-0.0012973 M_{z}+1.1565 \text { for } 5.3<=M_{z}<=9.0 \text {, }
\end{aligned}
$$


and

$$
\begin{aligned}
& v=0.002709 M_{z}^{2}-0.05645 M_{z}+1.2778 \text { for }-1<=M_{z}<=1.0, \\
& v=-0.0014881 M_{z}^{3}-0.0213937 M_{z}^{2}-0.1382798 M_{z} \\
& +1.3425 \text { for } 1<=M_{z}<=5.3 \text {, } \\
& v=-0.0012973 M_{z}+1.1565 \text { for } 5.3<=M_{z}<=9.0 \text {. }
\end{aligned}
$$

\section{Step 2 cost function}

This cost function is for searching the additional geoacoustic and scattering parameters such as $\sigma_{s}, \sigma_{2}$, and $\delta$ through a model-data fitting process. Two options are provided for comparison. Option 1 has been developed in this thesis, while option 2 has been taken from existing literature.

\section{Option 1}

The cost function $O_{1}$ is based on the difference between measured time series data $y_{\text {meas }}\left(t_{k}\right)$ and modeled time series data $y_{\text {mod }}\left(t_{k}\right)$ (envelopes of time series) at each time sample $t_{k}$. First, the difference between modeled and measured envelopes are computed at each time step,

$$
\Delta\left(t_{k}, \overline{\mathbf{M}}\right)=\left[y_{\text {mea }}\left(t_{k}\right)-y_{\text {mod }}\left(t_{k}\right)\right]^{2}
$$

where, $\overrightarrow{\mathbf{M}}$ is the vector of search space parameters. 
The optimal parameter set resulting from the model-data fit for each time step $t_{k}$ is,

$$
O\left(t_{k}, \overrightarrow{\mathbf{M}}_{k}\right)=\operatorname{argmin}\left(\Delta\left(t_{k}, \overrightarrow{\mathbf{M}}\right)\right)
$$

where $\overrightarrow{\mathbf{M}}_{k}$ is the best set of parameters at time step $t_{k}$. The optimal set for option 1 for the entire envelope matching is then,

$$
O_{1}=\frac{1}{K} \sum_{k} O\left(t_{k}, \overrightarrow{\mathbf{M}}_{k}\right)
$$

where $K$ is total number of time samples considered.

\section{Option 2}

The second option uses the cost function based on a normalized difference over the entire measured and modeled time series envelopes. It is described in the literature [20] as follows,

$$
E_{2}(\overrightarrow{\mathbf{M}})=\frac{1}{\sum_{k}\left[\left(y_{\text {meas }}^{2}\left(t_{k}\right)+y_{\text {mod }}^{2}\left(t_{k}\right)\right]\right.} \sum_{k}\left[y_{\text {meas }}\left(t_{k}\right)-y_{\text {mod }}\left(t_{k}\right)\right]^{2}
$$

The optimal set resulting from the model-data fit for each average envelope is,

$$
O_{2}=\operatorname{argmin}\left(E_{2}(\overrightarrow{\mathbf{M}})\right)
$$

\subsubsection{Search Algorithm}

The estimation of bottom parameters from the model-data matching process is complicated due to the possibility of a large number of good fits in the multidimensional search space. However, in some cases, the values found from optimization may not have a physical meaning. Therefore, it is necessary to establish constraints for each search space. Several 
approaches are described in the literature such as in [1]. In this paper, a two step search approach is proposed. Before the fitting procedures are applied, an estimate of the bottom reflection coefficient is done and used in step one to obtain grain size as one of the inputs to the model. The detailed algorithm for this is not described here but the coefficient is approximated from the measured data. Once the reflection coefficient is estimated, the mean grain size can be calculated. The relationship between the mean grain size, the reflection coefficient and the sediment impedance is described in [20]. The mean grain size is obtained by minimizing the cost function by equation (4.35). Instead of searching all five parameters at the same time by fitting the model to the measured data, two of the geoacoustic parameters, sound speed ratio and density ratio are approximated first through the mean grain size. The spectral strength parameter $W_{2}$ is also computed from the mean grain size. The second step is to obtain the three other unknown parameters by optimizing the model to the measured data. An exhaustive search algorithm is applied for this optimization process. The search range for each parameter is determined by using the mean grain size and Table 2 in [4] as a constraint. The tests with synthetic data have shown that by breaking down the optimization process into two steps the computation is more efficient and the estimation yields better results.

In the second step, the cost function (4.40) is minimized to optimize the agreement between model and measured data. With the consideration that the unknown parameters would be treated as the "random variable", instead of mapping the entire model to each ensemble of measured data to get one best estimated result for each measurement (as when 
using option 2 as the cost function), a point-to-point mapping procedure is performed (option 1 cost function). The final result using option 1 for each run produces the mean of the estimates found from the point-to-point procedure. For example, the model and one single measurement has the same length (500 samples) as the measured time series. The pointto-point mapping procedure will minimize the cost function at every single point (time sample) and will result in 500 best combination of estimates. The mean of these 500 estimates will be taken as the final result for this run. In this way, some of the smaller values in the time series that are primarily due to scattering are not dominated by the larger parts of the curve dominated by the geoacoustic parameters.

\subsubsection{Testing the optimization with synthetic data}

Synthetic data was generated with known seafloor parameters which were selected from Table.2 in [4] with additive white noise of about $-10 \mathrm{~dB}$. One hundred data sets (packets) were generated. Figures from 4.7 to 4.14 illustrate the results of the model-synthetic data fit using option 1 , cost function $O_{1}$. 


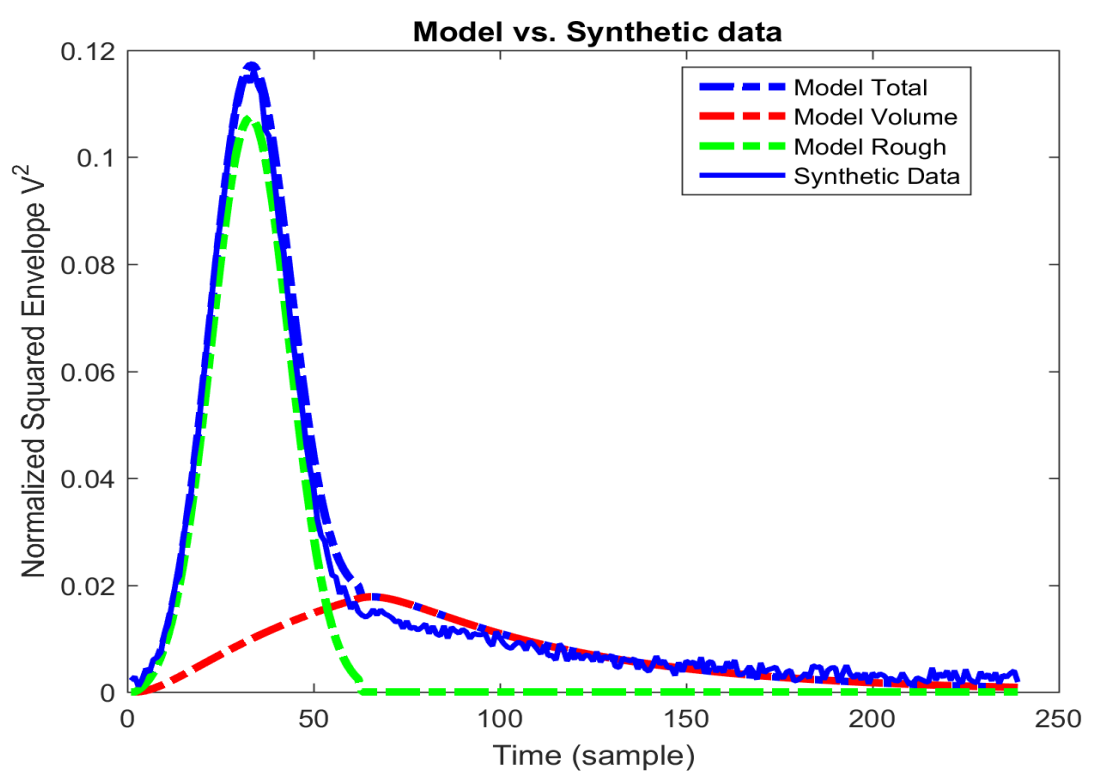

Figure 4.7: Best fit model vs. synthetic data.

The Fig 4.7 shows the agreement between the best fit, modeled signal and simulated signal. The estimated values found from the mapping process was fed back to the model to generate the plots of the total field and its components (interface and volume scattering). The synthetic data is shown as the solid blue line, the interface scattering contribution is shown as the green dashed line, the volume scattering component as the red dashed line, and the total modeled signal is the blue dashed line. Figures 4.8 to 4.14 present the distribution of parameters found from the optimization process. 


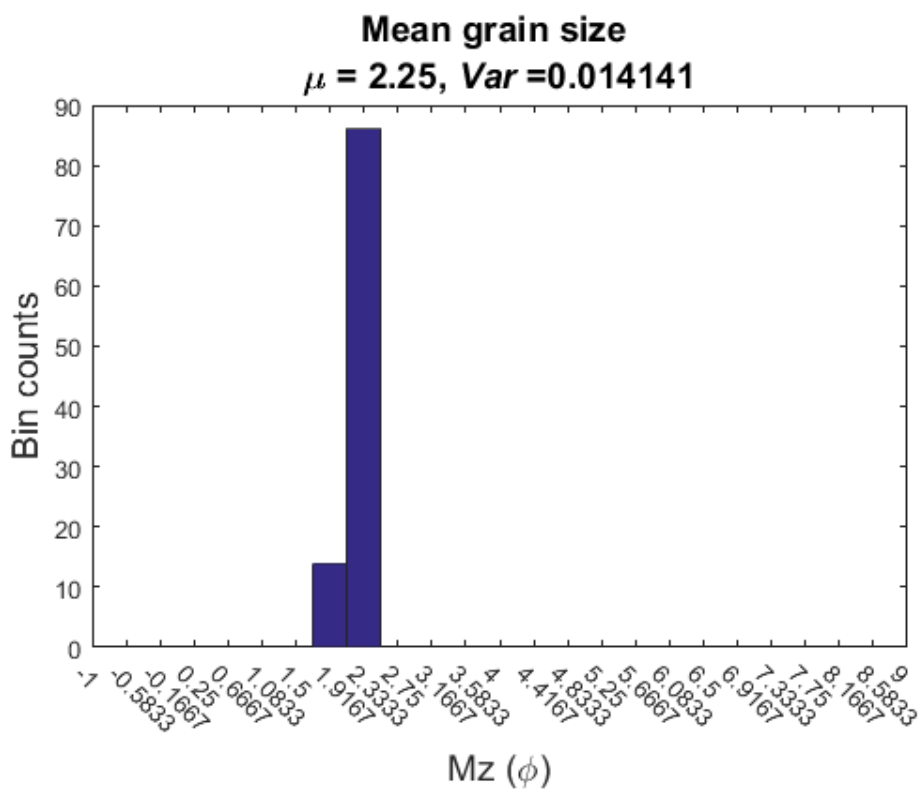

Figure 4.8: Estimated mean grain size $M_{z}$.

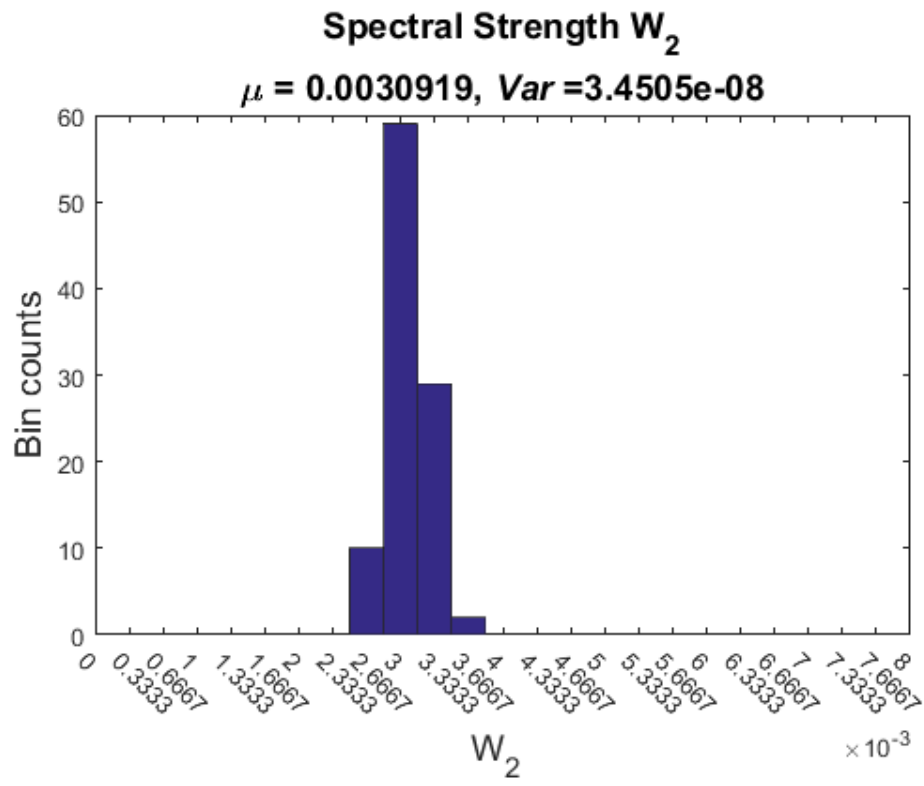

Figure 4.9: Estimated spectral strength $W_{2}$. 


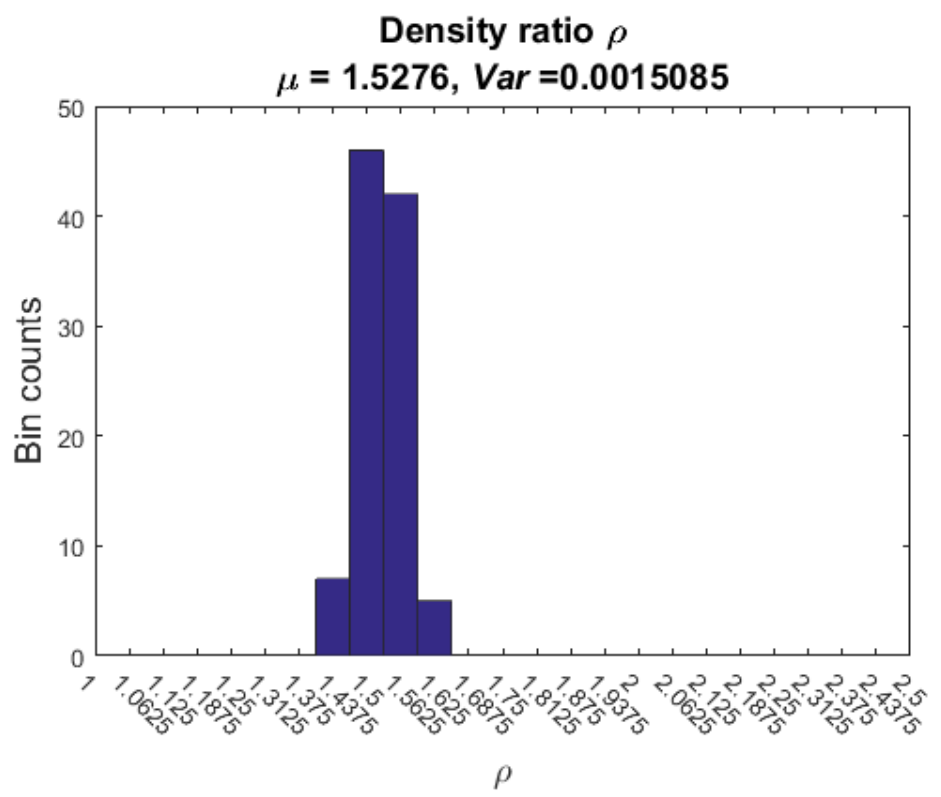

Figure 4.10: Density ratio $\rho$. True value is 1.5176 .

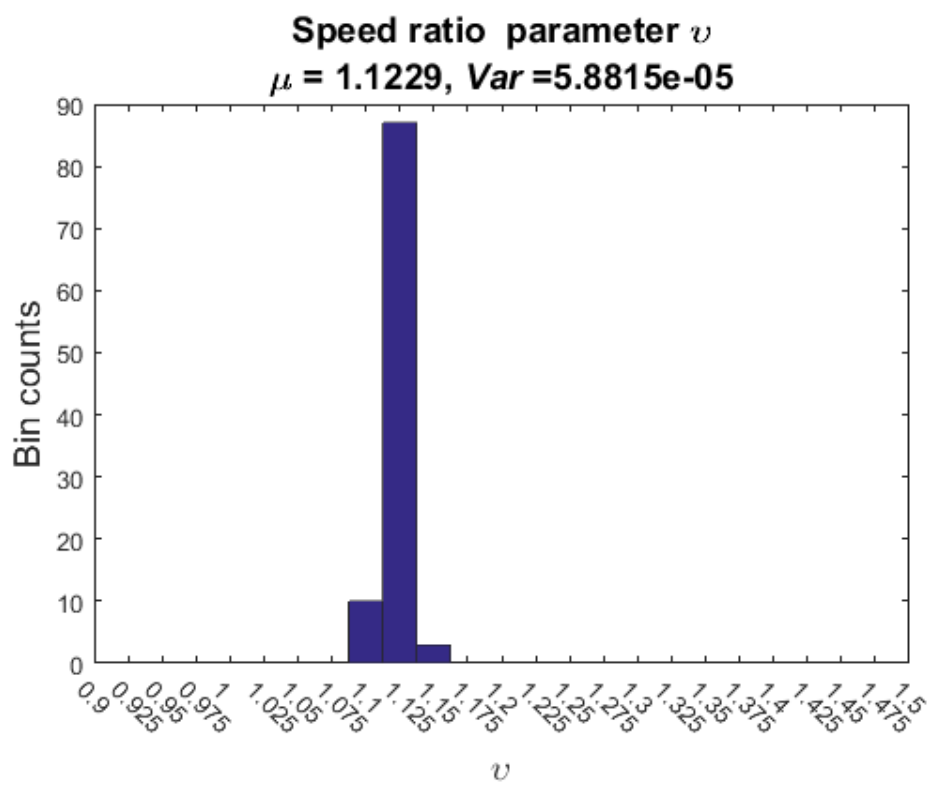

Figure 4.11: Speed ratio $v$. True value is 1.1660 . 


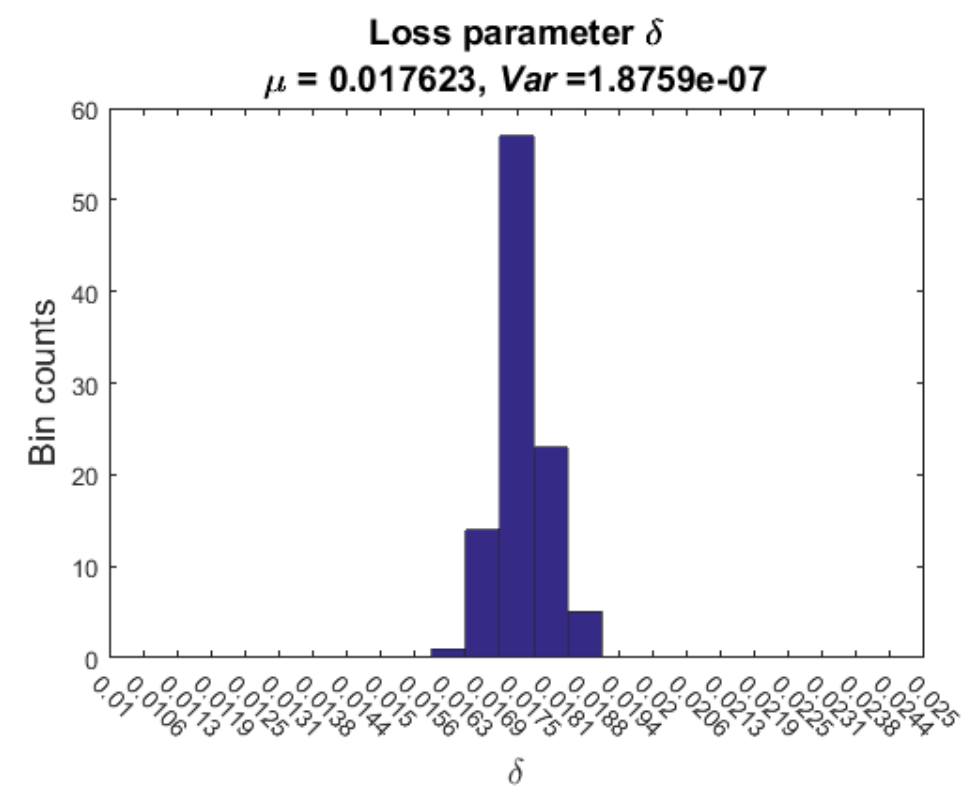

Figure 4.12: Loss parameter $\delta$. True value is 0.0183 .

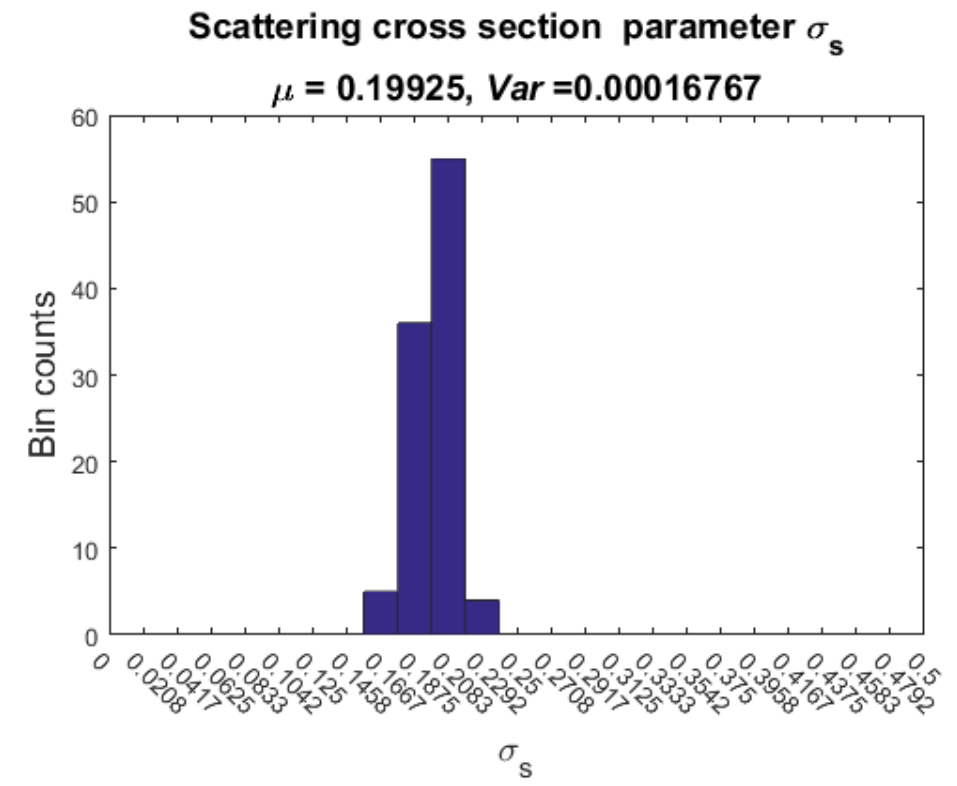

Figure 4.13: Interface scattering cross section $\sigma_{s}$. True value is 0.2097 


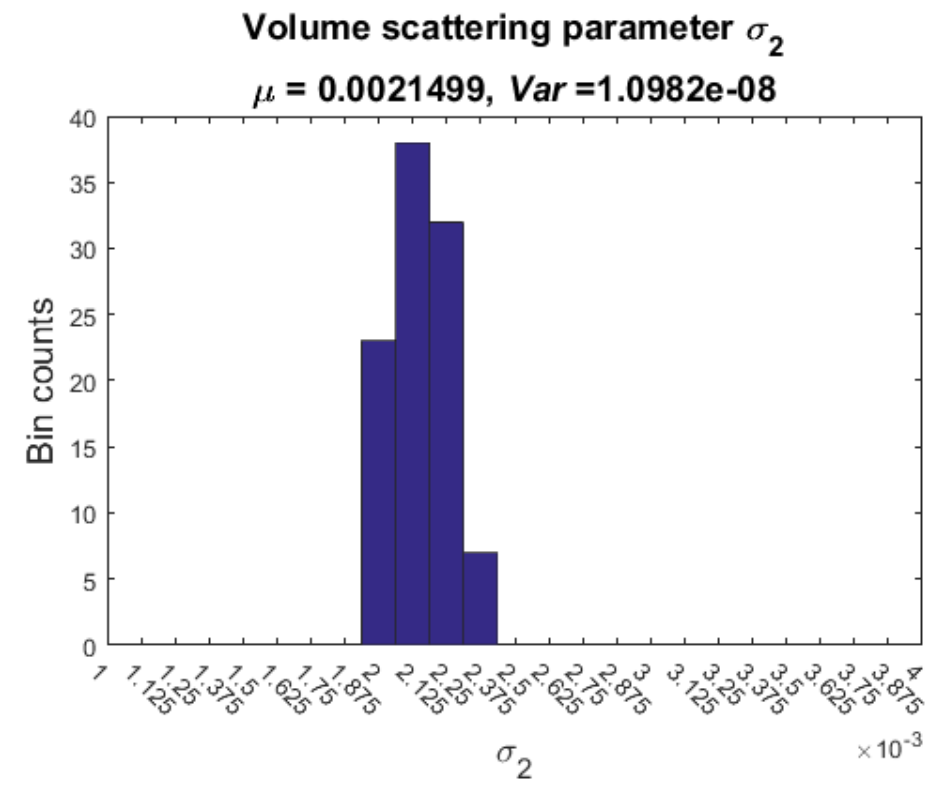

Figure 4.14: Volume scattering parameter $\sigma_{2}$. True value is 0.0021 .

\begin{tabular}{llllllll}
\hline Parameter: & $M_{z}(\phi)$ & $W_{2}\left(\mathrm{~cm}^{4}\right)$ & $\rho(-)$ & $v(-)$ & $\delta(-)$ & $\sigma_{s}$ & $\sigma_{2}(-)$ \\
\hline Estimates: & 2.25 & 0.0031 & 1.5276 & 1.229 & 0.0176 & 0.1992 & 0.0021 \\
True: & - & - & 1.5162 & 1.1660 & 0.0183 & 0.2097 & 0.0021 \\
& & & & & & & \\
Error (\%): & - & - & .75 & 3.7 & 3.82 & 5.01 & 0 \\
\hline
\end{tabular}

Table 4.2: Summary of test with synthetic data

Note that mean grain size $M_{z}$ and spectral strength $W_{2}$ were not the inputs of the model, so there is no available true value for comparison. The error is due to the noise added to the signal. 


\section{Chapter 5}

\section{Applying the model to measured data}

One set of experimental data was collected by the Applied Physics Laboratory at the University of Washington (APL-UW) along the Main Reverberation Track of the TREX13 experiment. Another set of data was collected by the Northwest Electromagnetics and Acoustics Research Laboratory (NEAR Lab) and has been used for testing with the model. Where it is required, the pre-processing steps that are described in Section 5.1 were applied to these data sets before they were matched to the modeled signal. Each envelope was computed from an average of 10 successive pings.

\subsection{Data processing}

Before comparing the measured and modeled signals, there were several pre-processing steps needed to take care of variations in the return signal due, for example, to differences in the signal arrival time caused by changes in bathymetry or slight movement of the source/receiver assembly. These steps are not necessary for synthetic data and they are described in the following paragraphs.

\subsubsection{Correcting the signal amplitude}

The signal amplitude needs to be corrected due to the spreading loss and attenuation in water column, the variation in bottom depth also need to be taken care if one uses the 
reference depth as an constant input to the model.

\subsubsection{Normalizing the data}

The measured data need to be normalized such that the source level is unity. This step is necessary as the model is predicting the shape of the normalized envelope. In practice a calibrated source level can not always be obtained, however it can be estimated by using multipath echoes in the acoustic data. For an example, if the acoustic data has multiple echos as shown in Fig. 5.1, the surface and bottom reflection losses can be obtained using the wave equation and principles of spreading and transmission loss as described in Section 2. Once the reflection losses are known, the source level can be approximated.

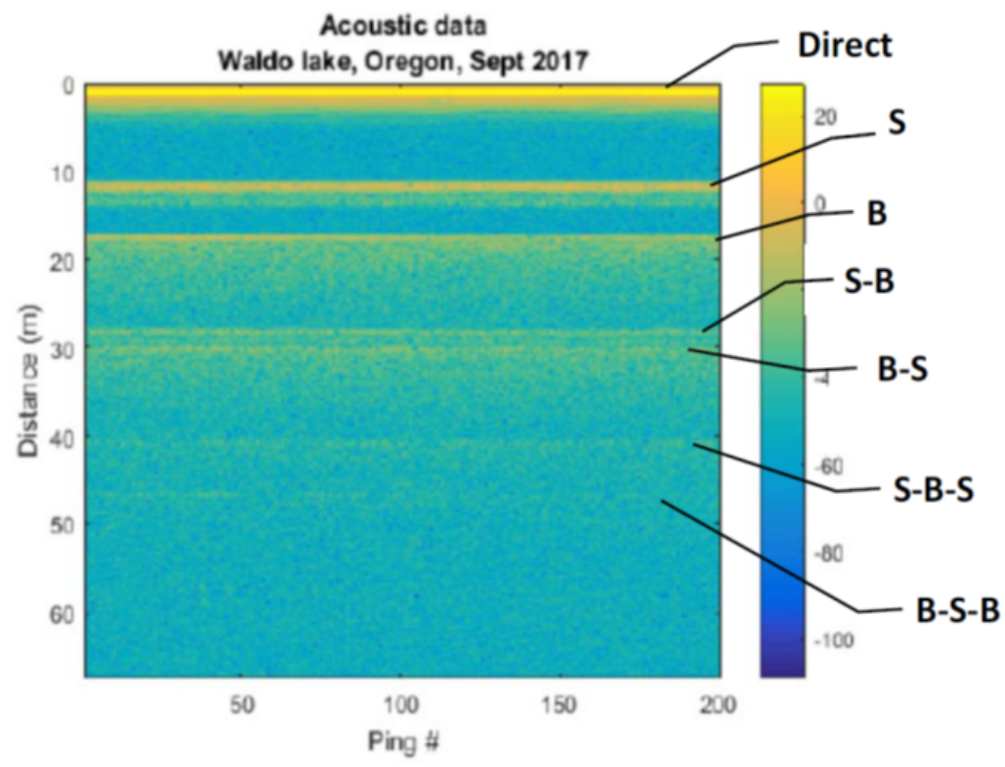

Figure 5.1: Acoustic data collected by NEAR lab at Waldo lake, Sept 2017

In Fig. 5.1, from the top, the second echo at about $11 \mathrm{~m}$ is the surface bounce, the third one is the bottom return followed by the surface -bottom and bottom-surface returns. 
The echo at 40 meter and 48 meter are surface-bottom-surface and bottom-surface-bottom bounces respectively. The diagram of multipaths is shown in Fig. 5.2 ,

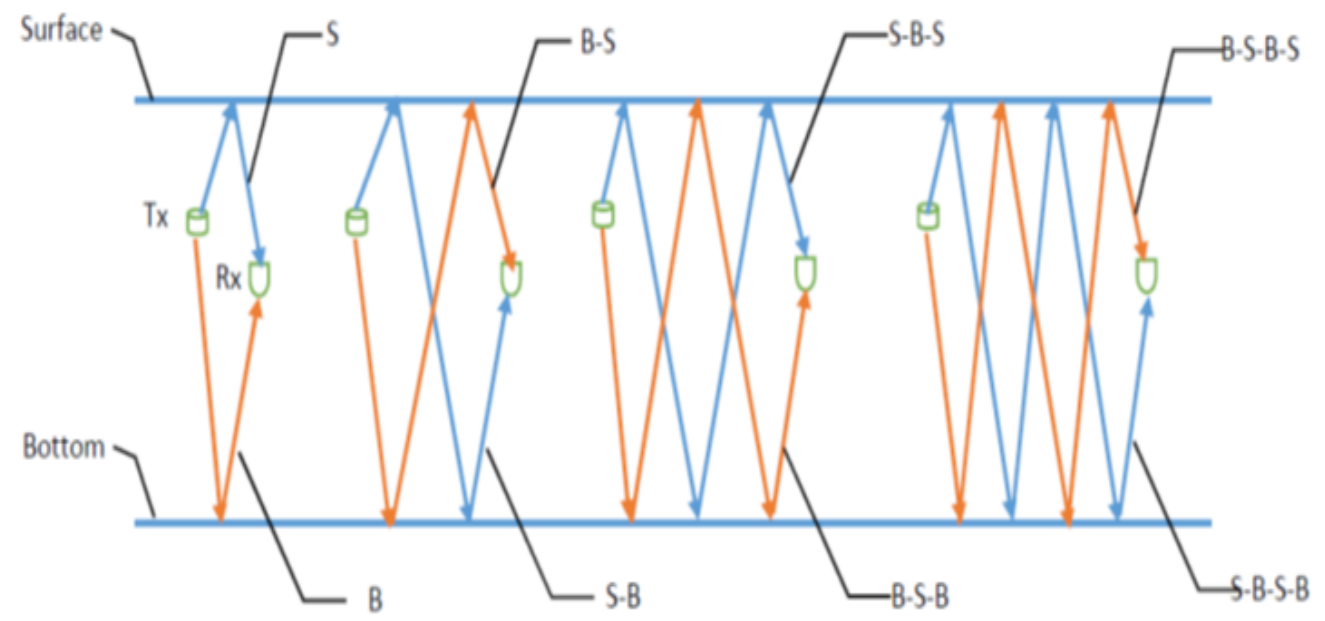

Figure 5.2: Diagram of multipaths data

Example of using sonar equation for estimation of bottom loss and surface loss is,

$$
B L=S+T L_{S}-B S-T L_{B S}
$$

and,

$$
S L=B S+T L_{B S}-S B S-T L_{S B S}
$$

where,

$$
\begin{array}{ll}
B L & : \text { Bottom loss } \\
S L & : \text { Surface loss } \\
S & : \text { Surface bounce energy level }
\end{array}
$$




$\begin{array}{ll}B S & : \text { Bottom-surface bounce energy level } \\ S B S & : \text { Surface-Bottom-Surface bounce energy level } \\ T L_{B S} \quad: \text { Transmission loss due to bottom-surface path } \\ T L S \quad: \text { Transmission loss due to surface path } \\ T L_{S B S} \quad: \text { Transmission loss due to surface-bottom-surface path }\end{array}$

Note that the energy levels are on $\mathrm{dB}$ scale.

\subsubsection{Aligning the signal}

Generally, the arrival time of signals will not be the same in different transmissions due to depth variations (bathymetry changes) or ship (source/receiver) movement. Aligning the return envelope is necessary before going to next step of data fitting. There are several methods for alignment, the way it was done here is to determine the approximate region of the return signals then find the peaks and shift all the signals to the same reference time.

\subsubsection{Averaging the signal}

Typically, the measured signal envelope will show variability in shape and amplitude due to a variety of factors not related to the parameters of interest such as noise. To reduce these variations the average envelope is computed from a certain $N$ number of pings. Note that this step will reduce the size of the data set. The modeled signal will be mapped with this average envelope. 


\subsection{Target and Reverberation Experiment 2013 (TREX13) data}

\subsubsection{Description of data set}

To perform the model data fitting process the SBES data as shown in Fig. 5.3 that was collected by the Applied Physics Laboratory at the University of Washington during the Target and Reverberation Experiment 2013 is considered. Although it is a bit lower frequency $(3 \mathrm{kHz})$ than the frequency the model is designed for (typically for frequencies $>10 \mathrm{kHz}$ ), it can still be used to illustrate the model matching procedures. The instrument used for experiment is a Bottom Backscatter SONAR (BSS), the sensors are an omnidirectional source (ITC-1007) and an omni-directional receiver (ITC-1032) mounted on a short bracket [21]. The transmitted pulse is a narrowband $3 \mathrm{kHz}$ signal. A Gaussian bandpass filter is applied to each ping. The data set consists of 500 pings is used for this optimization process. All the data processing steps have been applied to this data set. The echo returns are shown in Fig. 5.3. From the top of the figure, the second echo at about $5 \mathrm{~m}$ is the bottom bounce, the third one at $22 \mathrm{~m}$ is the surface return followed by the surface bottom and bottom-surface returns at $27 \mathrm{~m}$. The echo at $47 \mathrm{~m}$ is the bottom-surface-bottom bounce. 


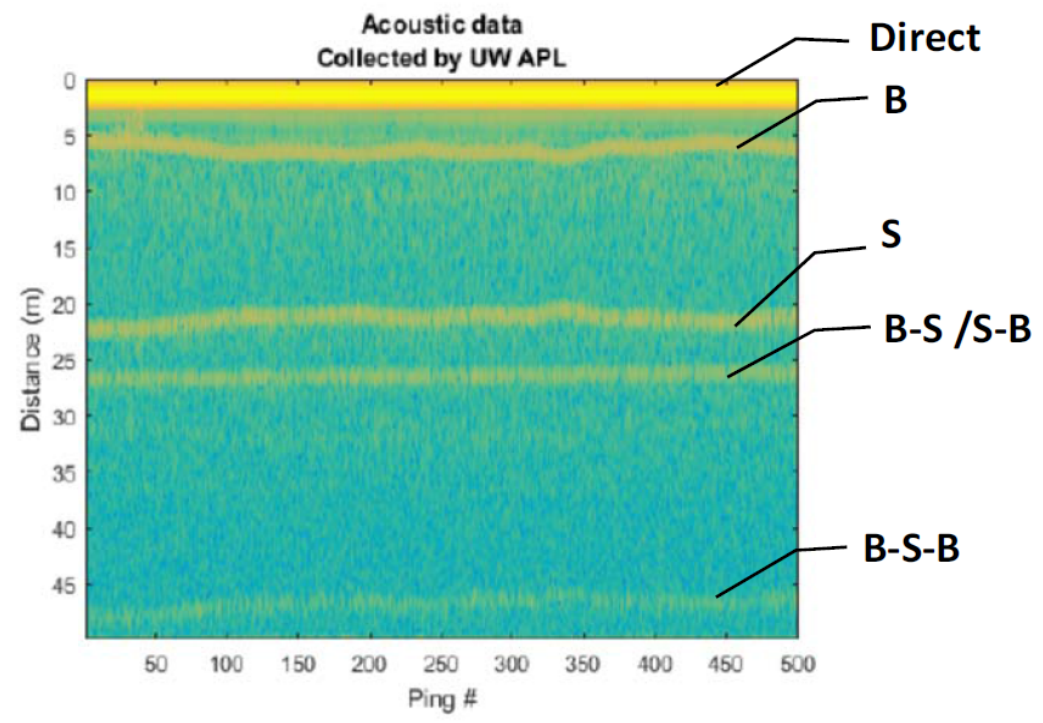

Figure 5.3: Acoustic data collected by Applied Phisycs Laboratory, University of Washington.

\subsubsection{Optimization results}

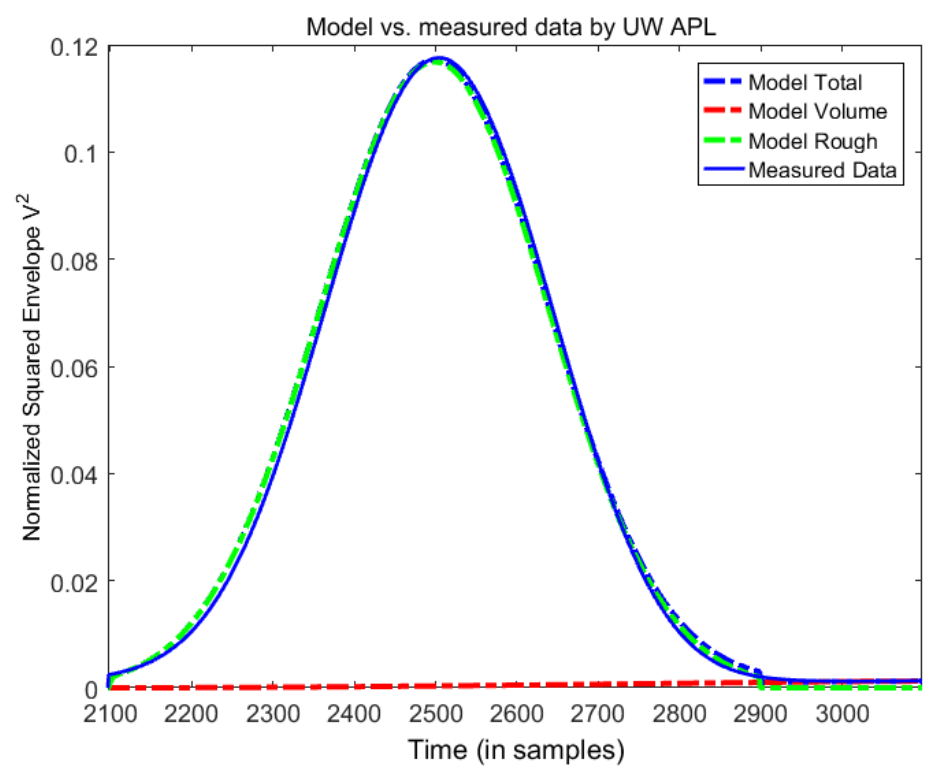

Figure 5.4: Model vs. measured data 
The blue solid line is the bottom return signal captured from measured data, the dashed red line and dashed green line are the volume scattering and interface scattering components of the modeled signal respectively. The dashed blue line is the total modeled signal. It can be seen that the model matches well with the measured signal.

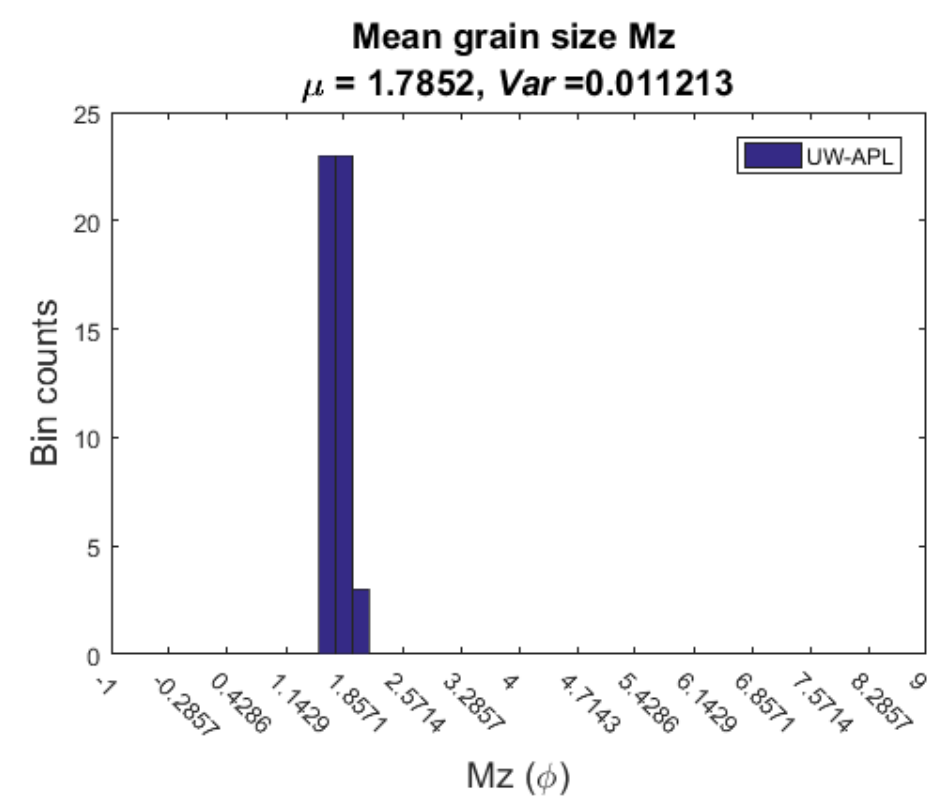

Figure 5.5: Estimated mean grain size $M_{z}$. 


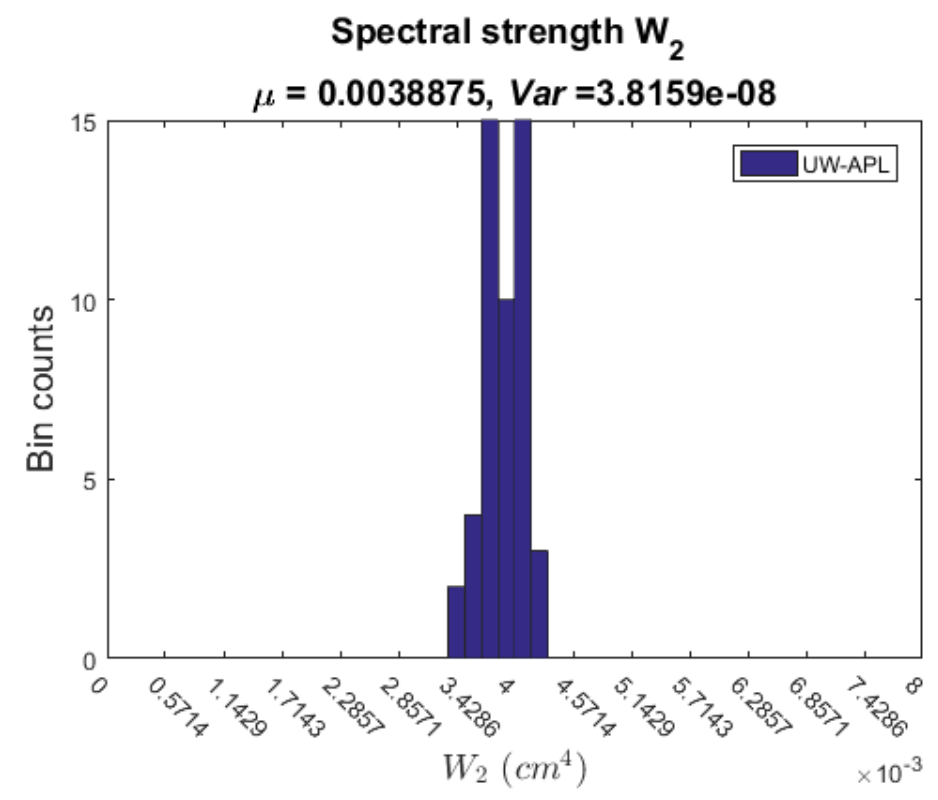

Figure 5.6: Estimated spectral strength $W_{2}$.

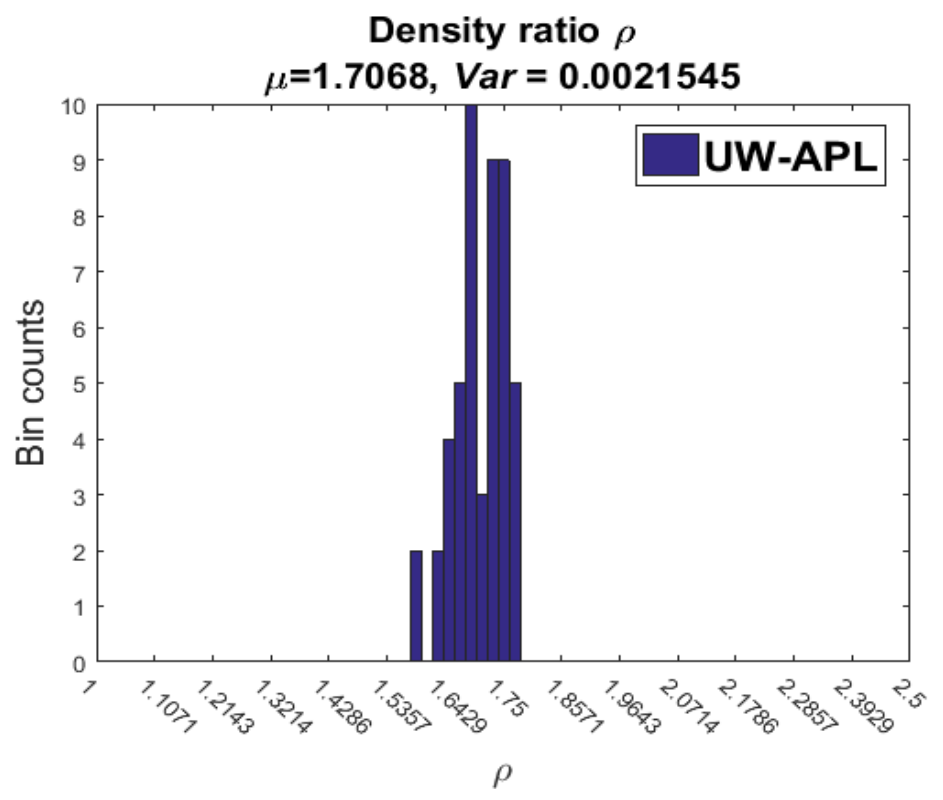

Figure 5.7: Density ratio $\rho$. 


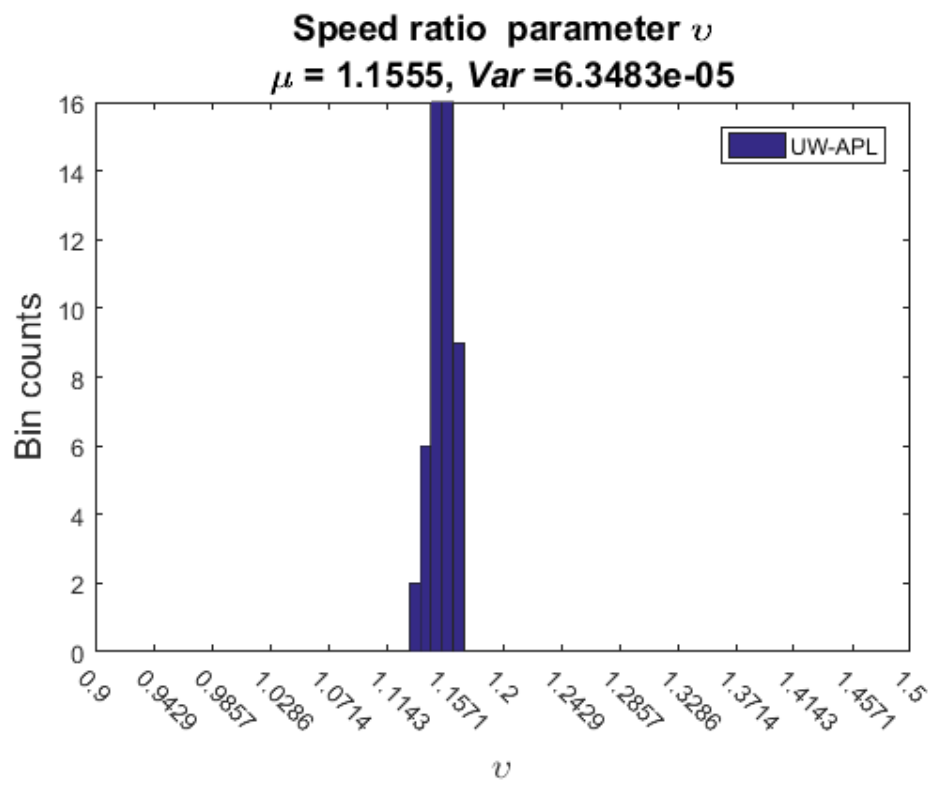

Figure 5.8: Speed ratio $v$.

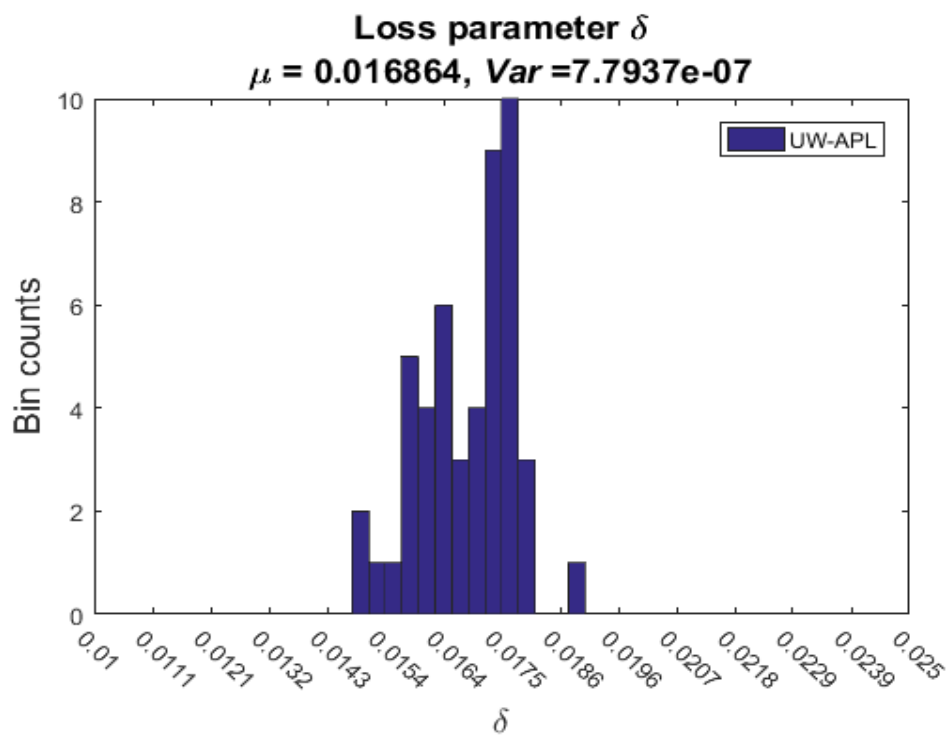

Figure 5.9: Loss parameter $\delta$. 


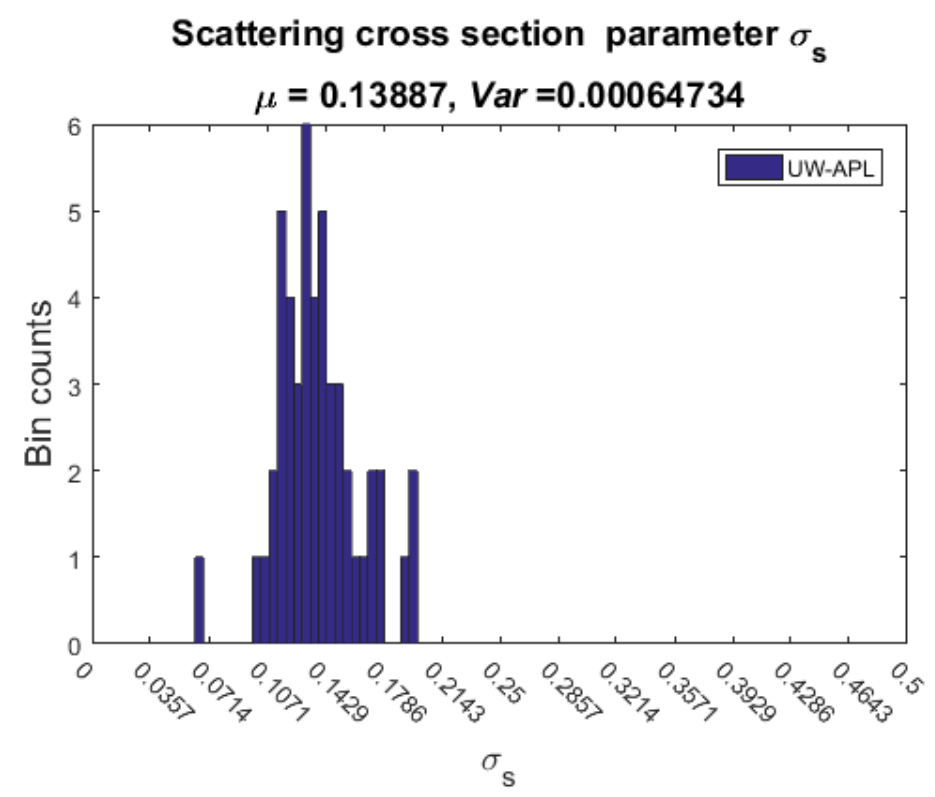

Figure 5.10: Interface scattering cross section $\sigma_{s}$.

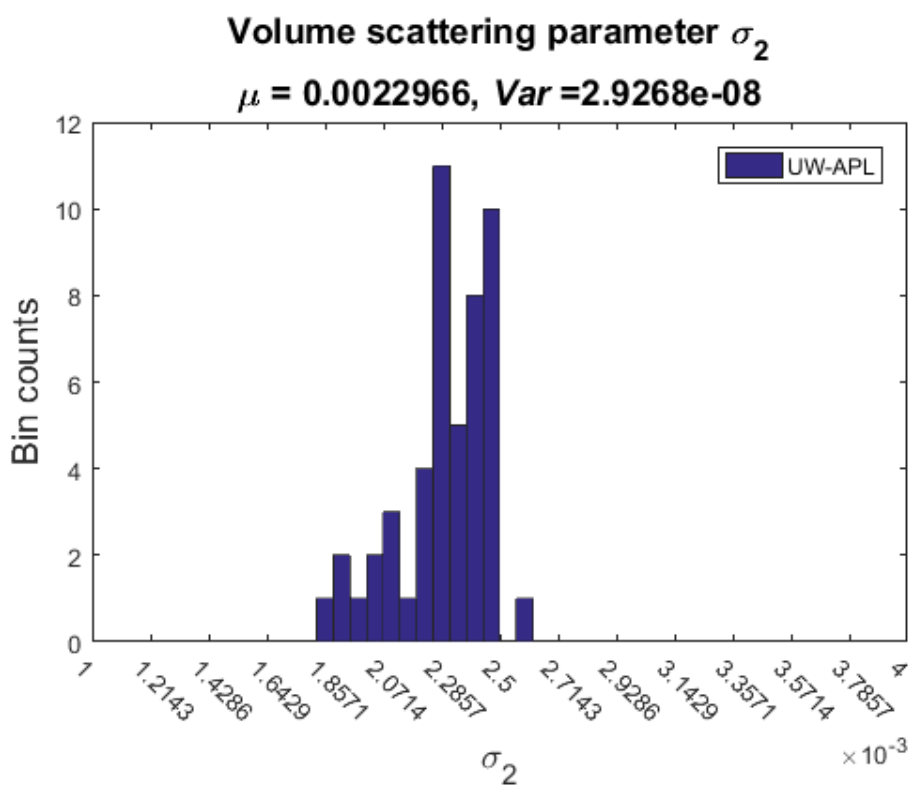

Figure 5.11: Volume scattering parameter $\sigma_{2}$. 


\subsection{Mapping with Waldo Lake data}

\subsubsection{Description of data set}

The data was collected by the author at Waldo lake, Oregon on September 2017. The instrument used for this experiments includes a conical transducer BII-7562/33IM that has a center frequency of $33 \mathrm{kHz}$, the hydrophone BII-7018, , the power amplifier BII-5020 and the data acquisition device NI USB-6281. All equipment is powered by batteries. The hydrophone was mounted a distance of 1 meter under the transducer on a rigid PCV pipe. The whole system was hung along side an inflatable boat and was deployed at a depth of 5 meters from the surface. The water column was approximately 14.5 meters. The boat was drifting slowly to avoid a big deviation of signal arrival times (due to bathymetry changes). A linear frequency modulated signal of $3 \mathrm{~ms}$ swept from 28 to $38 \mathrm{kHz}$ was transmitted

downwards every 1 second. A separation of 0.997 second between pings was used to make sure no overlap occurred between return signals and to have sufficient time for the system to acquire the multipath data. Two sets of 200 pings were collected.

\subsubsection{Optimization result:}

Presented here are the results from the fitting process. 


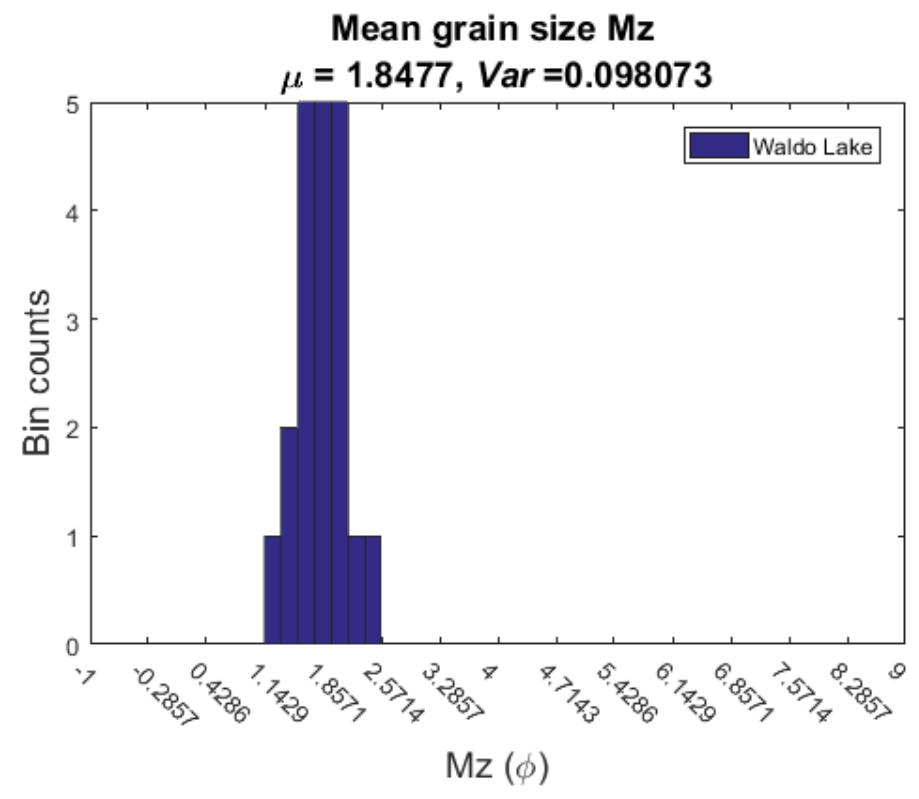

Figure 5.12: Estimated mean grain size $M_{z}$.

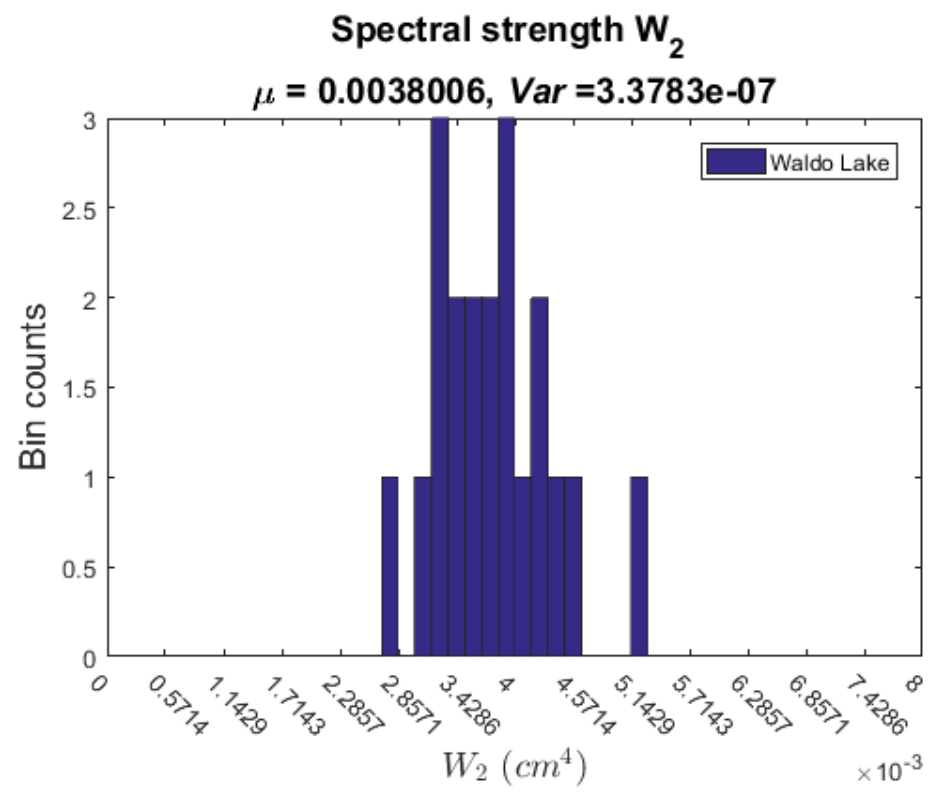

Figure 5.13: Estimated spectral strength $W_{2}$. 


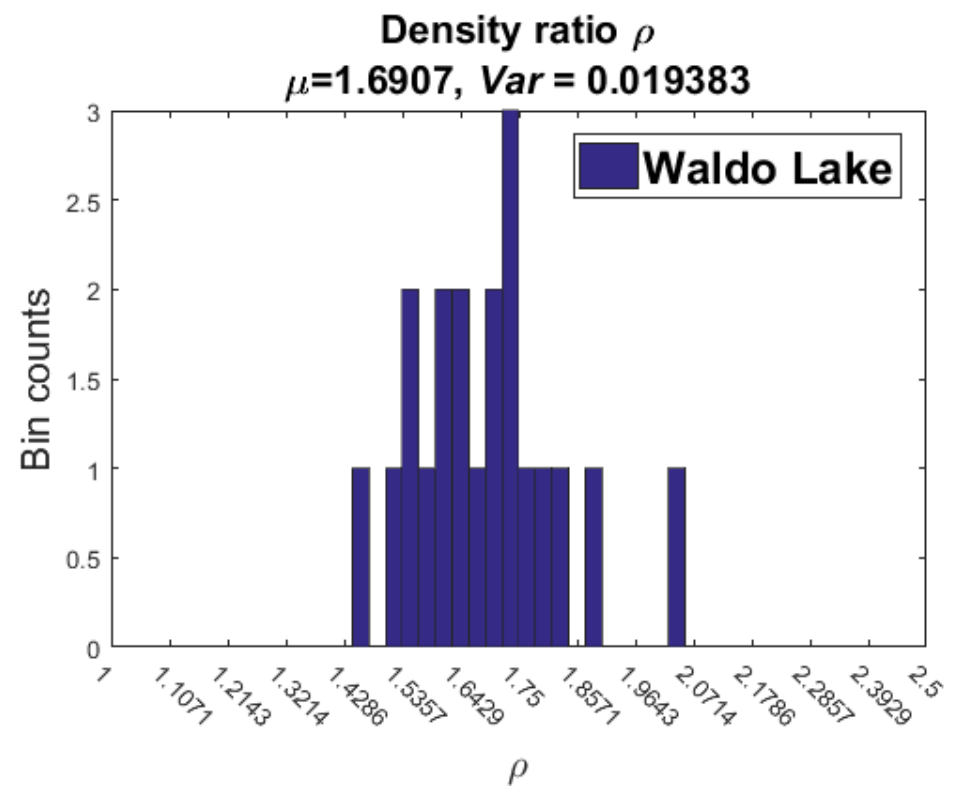

Figure 5.14: Density ratio $\rho$.

Sound speed ratio $v$ $\mu=1.1519$, Var $=5.614 \mathrm{e}-04$

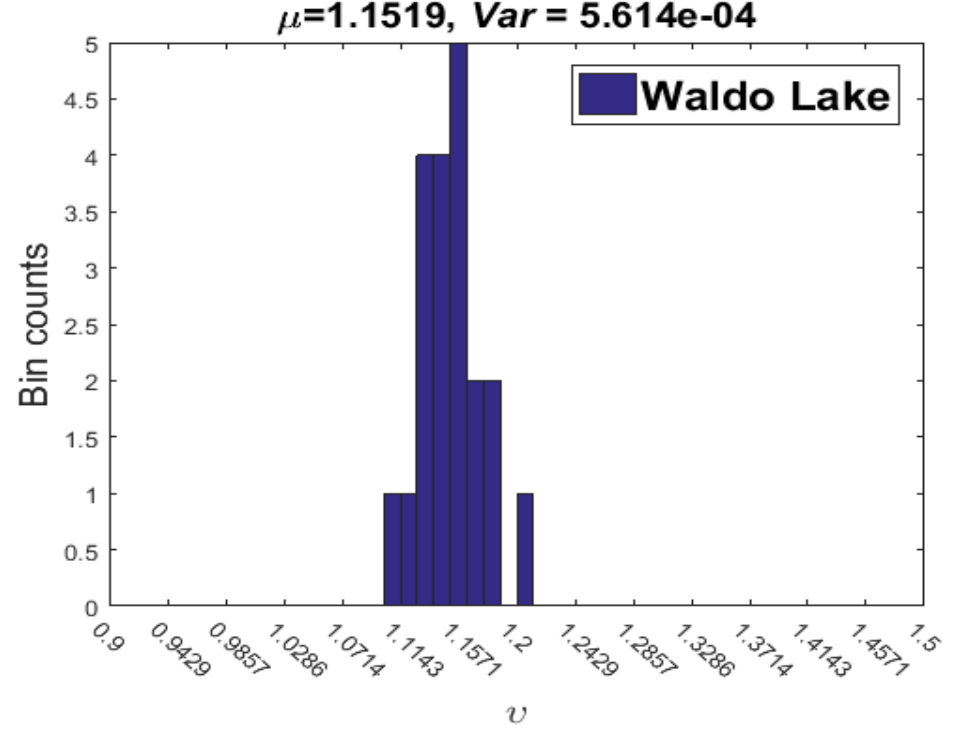

Figure 5.15: Speed ratio $v$. 


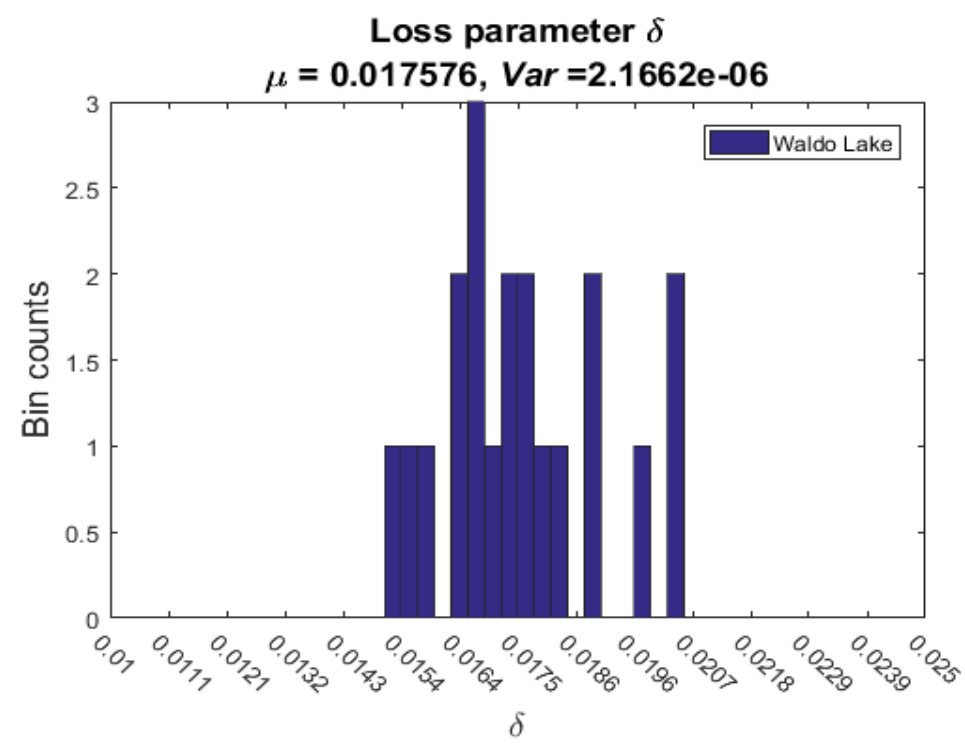

Figure 5.16: Loss parameter $\delta$.

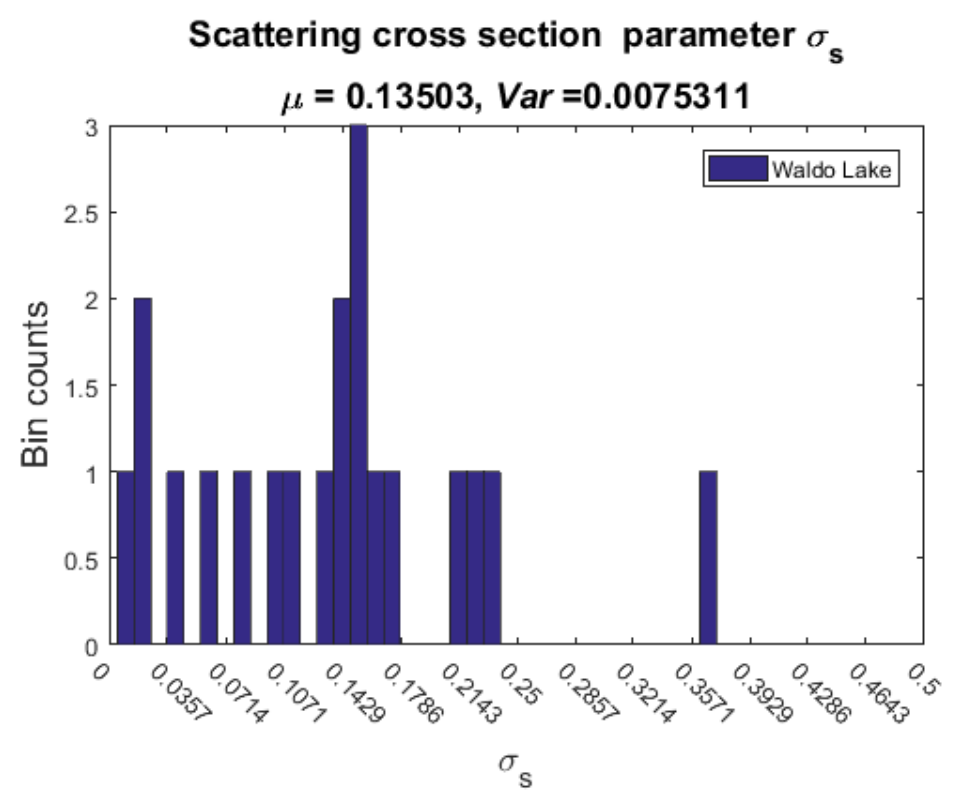

Figure 5.17: Interface scattering cross section $\sigma_{s}$. 


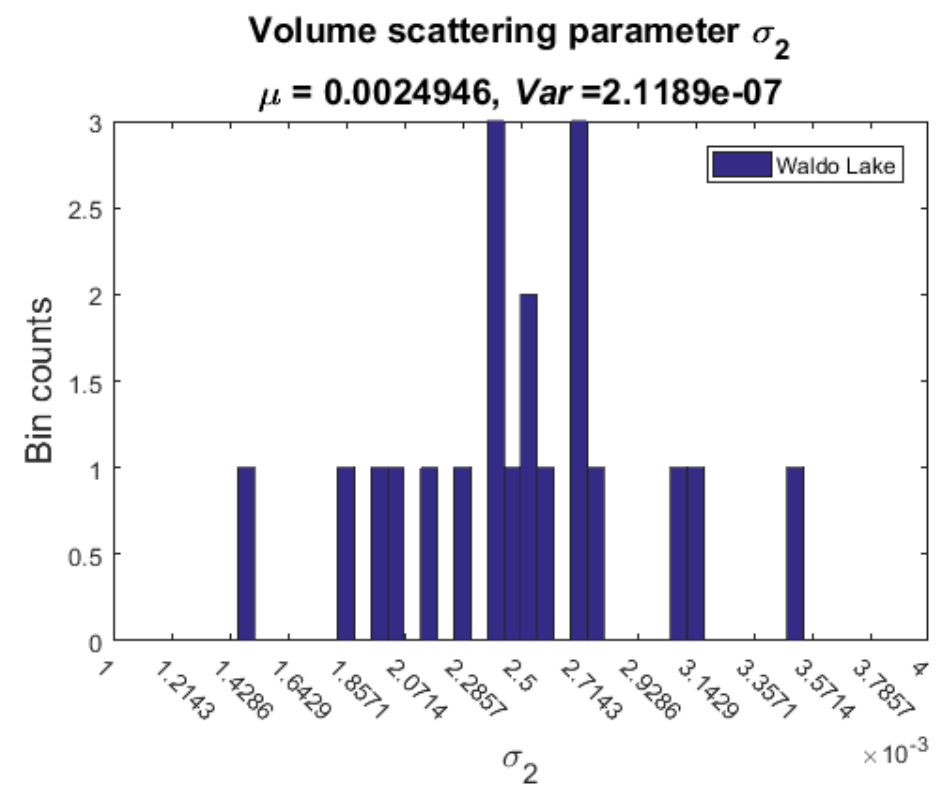

Figure 5.18: Volume scattering parameter $\sigma_{2}$.

\begin{tabular}{llllllll}
\hline Parameter: & $M_{z}(\phi)$ & $W_{2}\left(\mathrm{~cm}^{4}\right)$ & $\rho(-)$ & $v(-)$ & $\delta(-)$ & $\sigma_{2}(-)$ & $\sigma_{s}(-)$ \\
\hline UW-APL & 1.7852 & 0.0039 & 1.7068 & 1.1555 & 0.0169 & 0.0023 & 0.1389 \\
& & & & & & & \\
Waldo Lake & 1.8477 & 0.0038 & 1.6907 & 1.1519 & 0.0175 & 0.0025 & 0.1153 \\
\hline
\end{tabular}

Table 5.1: Summary of optimization results with measured data.

The Table. 5.1 presents the results from fitting process and the Table. 5.2 is the empirical data available in literature that matches closely to the mean grain size found from optimization. Note that no value of $\sigma_{s}$ is available from this literature.

\begin{tabular}{lcccccc}
\hline Sediment name: & $M_{z}(\phi)$ & $W_{2}\left(\mathrm{~cm}^{4}\right)$ & $\rho(-)$ & $v(-)$ & $\delta(-)$ & $\sigma_{2}(-)$ \\
\hline & & & & & & \\
Gravel Muddy sand & 1.0 & 0.00558 & 2.151 & 1.2241 & 0.01645 & 0.0020 \\
Medium sand & 1.5 & 0.00445 & 1.845 & 1.1782 & 0.01624 & 0.0020 \\
Muddy gravel & 2.0 & 0.00349 & 1.615 & 1.1396 & 0.01610 & 0.0020 \\
Fine, silty sand & 2.5 & 0.00272 & 1.451 & 1.1073 & 0.01602 & 0.0020 \\
Muddy sand & 3.0 & 0.00267 & 1.339 & 1.0800 & 0.01728 & 0.0020 \\
\hline
\end{tabular}

Table 5.2: Empirical data listed in [4]. 
The estimated results showed that the sediment in the areas where the data was taken is close to medium sand or muddy gravel. This is close to what was expected. 


\section{Chapter 6}

\section{Discussion}

A model fitting procedure has been developed to estimate geoacoustic and scattering parameters of the seabed from normal incident echo sounders. A new cost function was developed that appears to be better for estimating some of the less sensitive scattering parameters. Initial tests of the method use modeled data with additive noise as a synthetic data set. The results showed the optimization process was successful at estimating the relevant parameters. The method was also applied to measured data however since the true seabed is not known exactly it is difficult to assess the methods accuracy with these measured data sets.

Table. 5.1 and 5.2 present the mapping results and the empirical data. These are close to the estimated sediment characteristics in the regions where the measured data were collected. Having the value of $M_{z}$ found from step 1 of the optimization process, the empirical data can be adapted from Table.2 in [4]. It can be seen that the values of density ratio $\rho$, sound speed ratio $v$ and spectral strength $W_{2}$ resulting from the mapping process are slightly higher than the values that they are assumed to be if just the empirical data are used. It is consistent with some model-data fitting results in published literature (see [12] for example).

The use of this high frequency model with slightly lower frequency data from APL UW (the recommended frequency range is from $10 \mathrm{kHz}$ to $100 \mathrm{kHz}$ as per [4] and the mea- 
surements were $3 \mathrm{kHz}$ ) gives some promise of using the model-data fit with lower frequency acoustic data. The matching results with $28 \mathrm{kHz}$ data collected from Waldo Lake shows some supportive features such as the correlation between the estimated values and expected sediment parameters.

For future work, more comprehensive measurements are needed to further understand the physics of acoustic backscatter from seabed. Such measurements should be made over different areas and types of sediment. It would be perfect if the measurements could be done in the regions that have other empirical data available for comparison (e.g., core samples taken and analyzed).

The goal of this research is to study the possibility of using a model-based approach for seabed classification. The methodology presented in this report is still under development and needs improvement. However it can be said that it is feasible to employ a physics based method for seafloor classification without relying on ground truth for calibration. 


\section{Bibliography}

[1] Daniel D Sternlicht and Christian P de Moustier. Remote sensing of sediment characteristics by optimized echo-envelope matching. The Journal of the Acoustical Society of America, 114(5):2727-2743, 2003.

[2] Dimitri Alexandrou and D Pantzartzis. A methodology for acoustic seafloor classification. IEEE Journal of Oceanic Engineering, 18(2):81-86, 1993.

[3] Darrell Jackson and Michael Richardson. High-frequency seafloor acoustics. Springer Science \& Business Media, 2007.

[4] APL-UW High-Frequency Ocean Environmental. Acoustic models handbook. Applied Physics Laboratory, University of Washington, APL-UW TR, 9407, 1994.

[5] Waves-boundless physics. https://courses.lumenlearning.com/ boundless-physics/chapter/waves/.

[6] LJ Hamilton. Acoustic seabed classification systems. Technical report, DEFENCE SCIENCE AND TECHNOLOGY ORGANISATION VICTORIA (AUSTRALIA) AERONAUTICAL AND MARITIME RESEARCH LAB, 2001.

[7] Simon PR Greenstreet, Ian D Tuck, Gavin N Grewar, Eric Armstrong, David G Reid, and Peter J Wright. An assessment of the acoustic survey technique, roxann, as a means of mapping seabed habitat. ICES Journal of Marine Science, 54(5):939-959, 1997.

[8] Paul A Van Walree, Michael A Ainslie, and Dick G Simons. Mean grain size mapping with single-beam echo sounders. The Journal of the Acoustical Society of America, 120(5):2555-2566, 2006.

[9] R.J Urick. Principle of Underwater Sound. Peninsula Publishing, 1983.

[10] Martin Siderius. Array sensor processing. Lecture note, Sept 2016,Portland State University, Oregon.

[11] Daniel D Sternlicht and Christian P de Moustier. Time-dependent seafloor acoustic backscatter (10-100 khz). The journal of the acoustical society of America, 114(5):2709-2725, 2003.

[12] Darrell R Jackson and Kevin B Briggs. High-frequency bottom backscattering: Roughness versus sediment volume scattering. The Journal of the Acoustical Society of America, 92(2):962-977, 1992. 
[13] John T Anderson, V Holliday, RUDY Kloser, DAVID Reid, and YVAN Simard. Acoustic seabed classification of marine physical and biological landscapes. International Council for the Exploration of the Sea, 2007.

[14] Paul G von Szalay and Robert A McConnaughey. The effect of slope and vessel speed on the performance of a single beam acoustic seabed classification system. Fisheries Research, 56(1):99-112, 2002.

[15] Ali R Amiri-Simkooei, Mirjam Snellen, and Dick G Simons. Principal component analysis of single-beam echo-sounder signal features for seafloor classification. IEEE Journal of Oceanic Engineering, 36(2):259-272, 2011.

[16] LJ Hamilton, PJ Mulhearn, and R Poeckert. Comparison of roxann and qtc-view acoustic bottom classification system performance for the cairns area, great barrier reef, australia. Continental Shelf Research, 19(12):1577-1597, 1999.

[17] BT Prager, DA Caughey, and RH Poeckert. Bottom classification: operational results from qtc view. In OCEANS'95. MTS/IEEE. Challenges of Our Changing Global Environment. Conference Proceedings., volume 3, pages 1827-1835. IEEE, 1995.

[18] Kari E Ellingsen, John S Gray, and Erik Bjrnbom. Acoustic classification of seabed habitats using the qtc view system. ICES Journal of Marine Science, 59(4):825-835, 2002.

[19] Darrell R Jackson, Dale P Winebrenner, and Akira Ishimaru. Application of the composite roughness model to high-frequency bottom backscattering. The Journal of the Acoustical Society of America, 79(5):1410-1422, 1986.

[20] Mirjam Snellen, Kerstin Siemes, and Dick G Simons. Model-based sediment classification using single-beam echosounder signals. The Journal of the Acoustical Society of America, 129(5):2878-2888, 2011.

[21] DJ Tang and Todd Hefner. Bottom reflectivity along the main reverberation track of trex13. unpublished report.

[22] Darrell R Jackson, Kevin B Briggs, Kevin L Williams, and Michael D Richardson. Tests of models for high-frequency seafloor backscatter. IEEE journal of oceanic engineering, 21(4):458-470, 1996.

[23] Eric I Thorsos. The validity of the kirchhoff approximation for rough surface scattering using a gaussian roughness spectrum. The Journal of the Acoustical Society of America, 83(1):78-92, 1988.

[24] JH Stockhausen. Scattering from the volume of an inhomogeneous half-space. The Journal of the Acoustical Society of America, 35(11):1893-1893, 1963. 


\section{Appendix A}

\section{APL model parameterizations}

The geoacoustic inputs to the APL-UW model listed in Table. 4.1 are parameterized in terms of the bulk mean grain size $\mathrm{Mz}$ as follows,

Density ratio

Range : $1<=\rho<=3$

$$
\begin{aligned}
& \rho=0.007797 M_{z}^{2}-0.17057 M_{z}+2.3139 \text { for }-1<=M_{z}<=1.0 \text {, } \\
& \rho=-0.165406 M_{z}^{3}+0.2290201 M_{z}^{2}-1.1069031 M_{z} \\
& +3.0455 \text { for } 1<=M_{z}<=5.3 \text {, } \\
& \rho=-0.0012973 M_{z}+1.1565 \text { for } 5.3<=M_{z}<=9.0 \text {, }
\end{aligned}
$$

\section{Sound speed ratio}

$$
\begin{aligned}
& v=\frac{c_{2}}{c_{1}} \\
& v=0.002709 M_{z}^{2}-0.05645 M_{z}+1.2778 \text { for }-1<=M_{z}<=1.0 \\
& v=-0.0014881 M_{z}^{3}-0.0213937 M_{z}^{2}-0.1382798 M_{z} \\
& +1.3425 \text { for } 1<=M_{z}<=5.3 \\
& v=-0.0012973 M_{z}+1.1565 \text { for } 5.3<=M_{z}<=9.0
\end{aligned}
$$

\section{Loss parameter}

$$
\begin{aligned}
\delta & =\frac{k_{2 i}}{k_{2 r}}=\frac{\alpha_{2} v c_{1} \ln (10)}{40 \pi f} & & \\
\frac{\alpha_{2}}{f} & =0.4556 & & \text { for }-1<=M_{z}<=0 \\
& =0.4556+0.0245 M_{z} & & \text { for } 0<=M_{z}<=2.6 \\
& =0.1978+0.1245 M_{z} & & \text { for } 2.6<=M_{z}<=4.5 \\
& =8.0399-2.5228 M_{z}+0.20098 M_{z}^{2} & & \text { for } 4.5<=M_{z}<=6.0 \\
& =0.9431-0.2041 M_{z}+0.0117 M_{z}^{2} & & \text { for } 6.0<=M_{z}<=9.0
\end{aligned}
$$




\section{Volume parameter}

$\sigma_{2}$ tends to be independent of frequencies over $10-100 \mathrm{kHz}$ and $0.001<=\sigma_{2}<=0.005$ [22], [19]

$$
\begin{array}{lr}
\sigma_{2}=0.002 & \text { for }-1<=M_{z}<=5.5 \\
\sigma_{2}=0.001 & \text { for } 5.5<=M_{z}<=9.0
\end{array}
$$

Spectral exponent $\gamma$ is obtained from experimental power spectrum by changing the sign of spectral slope $2.4<=\gamma<=4$. If absence of measurement $\gamma=3.25$.

Spectral strength Range: $0<=W_{2}<=1$.

$$
\begin{aligned}
\frac{h}{h_{0}} & =\frac{2.03846-0.26923 M_{z}}{1+0.076923 M_{z}} & \text { for }-1<=M_{z}<=5.0 \\
\frac{h}{h_{0}} & =0 . .5 & \text { for } 5.0<=M_{z}<=9.0 \\
h_{0} & =1 \mathrm{~cm} & \text { is reference length } \\
W_{2} & =0.00207 h^{2} h_{0}^{2} & \text { For } \gamma=3.25
\end{aligned}
$$




\section{Appendix B}

\section{The roughness scattering approximation}

Roughness scattering cross section can be modeled in three ways as listed below and the method chosen depends on the situaion [3], [23]:

1. The Kirchhoff approximation, valid for smooth and moderately rough seabed interfaces with grazing angle near vertical $\left(90^{\circ}\right)$.

2. The composite roughness approximation, valid for smooth and moderately rough seabed interfaces with grazing angles away from $90^{\circ}$.

3. The large scale roughness approximation, to account for scattering strengths for gravel and rock bottom that are relatively large and imply roughness parameters fall outside Kirchhoff approximation.

The two most widely used approximations for scattering by seafloor roughness are smallroughness perturbation and Kirchhoff approximation. Each has its own separate domain of validity, for the scattering near the specular direction, the Kirchhoff works better [23]. The object of primary interest is scattering cross section . It is a function of the angles defining the direction from source to the scattering region and from scattering region to the receiver. In term of wave-number, the direction from the source to the scattering region and from 
scattering region to the receiver can be specified as

$$
\begin{aligned}
& \mathbf{k}_{i}=k_{w}\left(\mathbf{e}_{x} \cos \theta_{i} \cos \phi_{i}+\mathbf{e}_{y} \cos \theta_{i} \sin \phi_{i}-\mathbf{e}_{z} \sin \theta_{i}\right) \\
& \mathbf{k}_{s}=k_{w}\left(\mathbf{e}_{x} \cos \theta_{s} \cos \phi_{s}+\mathbf{e}_{y} \cos \theta_{s} \sin \phi_{s}+\mathbf{e}_{z} \sin \theta_{s}\right)
\end{aligned}
$$

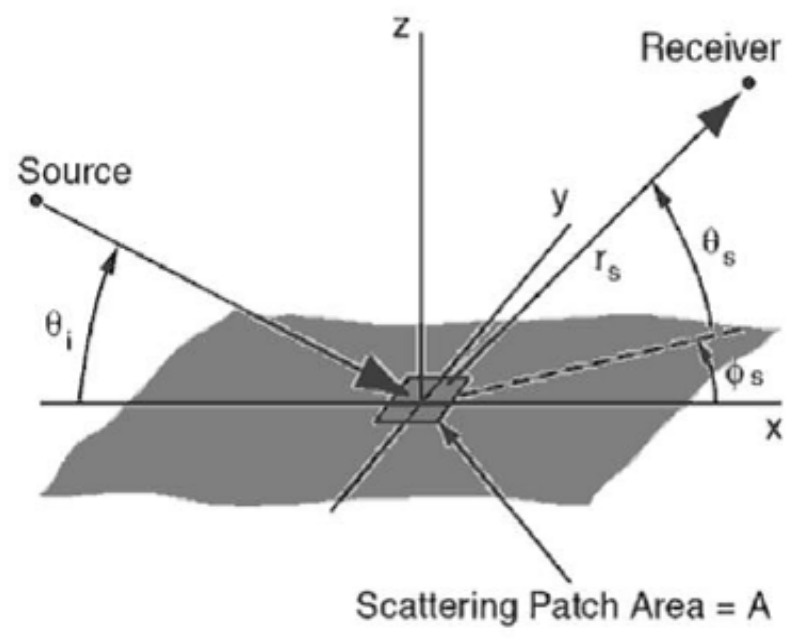

Figure B.1: Definition of angular coordinates used in treating reflection and scattering

Here, $k_{w}$ is the wavenumber in water, the incident direction is denoted as $\mathbf{k}_{i}$ and the direction towards receiver is $\mathbf{k}_{s} . \mathbf{e}_{x}, \mathbf{e}_{y} \mathbf{e}_{z}$ are the unit vector in $\mathrm{x}, \mathrm{y}$ and $\mathrm{z}$ direction respectively. The angles used here are defined in Fig. 2.4 where it is noted that one may set $\phi_{i}=0$ without loss of generality if seafloor is isotropic. All the components of the wave vectors can not be specified independently, as the magnitude must be $k+w$. Thus, the following horizontal horizontal components of the wave vectors are sufficient to define the 
incident and scattering directions:

$$
\begin{gathered}
\mathbf{K}_{i}=k_{w}\left(\mathbf{e}_{x} \cos \theta_{i} \cos \phi_{i}+\mathbf{e}_{y} \cos \theta_{i} \sin \phi_{i}\right) \\
\mathbf{K}_{s}=k_{w}\left(\mathbf{e}_{x} \cos \theta_{s} \cos \phi_{s}+\mathbf{e}_{y} \cos \theta_{s} \sin \phi_{s}\right)
\end{gathered}
$$

It is convenient to define the wave vector difference between the incident and scattering directions

$$
\Delta \mathbf{k}=\mathbf{k}_{s}-\mathbf{k}_{i}
$$

$$
\Delta \mathbf{K}=\mathbf{K}_{s}-\mathbf{K}_{i}
$$

and for one dimensional

$$
\Delta k_{z}=k_{s z}-k_{i z}
$$

Note that:

$$
\Delta k^{2}=\Delta K^{2}+\Delta k_{z}^{2}
$$

In this study, the Kirchhoff approximation is applied. The simplified form of backscattering ccross section in high frequency limit reduced to,

$$
\sigma_{\text {sur }}=\frac{1}{4}\left|V_{w w}\left(\theta_{i s}\right)\right|^{2} \frac{\Delta k^{4}}{\Delta k_{z}^{4}} p_{s}(\mathbf{s}) .
$$


where $V_{w w}$ is the seabed reflection coefficient for a flat interface as a function of grazing angle $\theta_{i s}$ where

$$
\theta_{i s}=\sin ^{-1}\left(\frac{\Delta k}{2 k_{w}}\right)
$$

$p_{s}(s)$ is the bivariate Gaussian probability density function for interface slope,

$$
p_{s}(\mathbf{s})=\frac{1}{2 \pi\left\|\mathbf{B}_{s}\right\|^{\frac{1}{2}}} e^{\left(-\frac{1}{2} s^{T} \mathbf{B}_{s}^{-1} \mathbf{s}\right)}
$$

where $s$ is defined as,

$$
\mathbf{s}=\left[\frac{\Delta K_{x}}{\Delta k_{z}} ; \frac{\Delta K_{y}}{\Delta k_{z}}\right]
$$

This column matrix contains the $x$-and $y$-components of slope corresponding to specular reflection and $\mathbf{B}_{\mathbf{s}}$ is co-variance matrix for slope with determinant $\left\|\mathbf{B}_{\mathbf{s}}\right\|$.

$$
\mathbf{B}_{\mathbf{s}}=\left(\begin{array}{cc}
<\left(\frac{d f}{d x}\right)^{2}> & <\frac{d f}{d x}><\frac{d f}{d y}> \\
<\frac{d f}{d x}><\frac{d f}{d y}> & <\left(\frac{d f}{d y}\right)^{2}>
\end{array}\right)
$$

where $f(\mathbf{R})$ is "interface relief function" with zero mean and quantity $h=<f^{2}(\mathbf{R})>$ is referred as $\mathrm{RMS}$ roughness for $\mathbf{R}=(x, y)$

In the isotropic case which is considered in this research, the slope covariance matrix $\mathbf{B}_{\mathrm{s}}$ is equal to unit matrix multiplied by constant and the eq. (B.9) becomes,

$$
\sigma_{\text {sur }}=\frac{\left|V_{w w}\left(\theta_{i s}\right)\right|^{2}}{8 \pi \sigma_{s}^{2}} \frac{\Delta k^{4}}{\Delta k_{z}^{4}} e^{\left(-\frac{\Delta K^{2}}{2 \sigma_{s}^{2} \Delta k_{z}^{2}}\right)}
$$


where

$$
\sigma_{s}=<\left(\frac{d f}{d x}\right)^{2}>=<\left(\frac{d f}{d y}\right)^{2}>
$$

is the RMS slope for a 1D track. In terms of an isotropic spectrum,

$$
\sigma_{s}^{2}=\pi \int K^{3} W(K) d K
$$

where, $W(k)$ is the surface spatial spectrum. The high frequency Kirchhoff approximation is most appropriately applied to backscatter from seafloor with isotropic roughness statistic, for that the equation $(\mathrm{B} .14)$ takes the simplified form,

$$
\sigma_{\text {sur }}=\frac{\left|V_{w w}\left(90^{0}\right)\right|^{2}}{8 \pi \sigma_{s}^{2} \sin ^{4} \theta_{i}} e^{-\frac{\cot ^{2} \theta_{i}}{2 \sigma_{s}^{2}}}
$$

The notation that has been used in above subsection is summarized below:

subscripts $i$ and $s$ denote for incident and scattering quaatities

$k_{w} \quad$ wave-number in seawater

$k_{w}^{\prime \prime} \quad$ imaginary part of wave-number in seawater

$\theta_{i s} \quad$ local grazing angle for specular reflection

$\theta_{s} \quad$ incident grazing angle

$V_{w w} \quad$ reflection coefficient for flat interface

$\Delta \mathrm{k} \quad$ magnitude of three dimensional wave vector difference 
$\Delta k_{z} \quad$ difference in vertical wave vector component

$\Delta \mathbf{K} \quad$ two-dimensional horizontal wave vector difference

$\Delta \mathbf{k} \quad$ two-dimensional horizontal wave vector difference

$\Delta K \quad$ magnitude of $\Delta \mathrm{K}$

$\Delta K_{x} \quad x$ component of $\Delta \mathbf{K}$

$\Delta K_{y} \quad y$ component of $\Delta \mathbf{K}$

K two dimensional horizontal wave number

K magnitude of two dimensional wave-number $\mathbf{K}$

$V_{w w}\left(90^{0}\right) \quad$ reflection coefficient of flat interface at near nadir

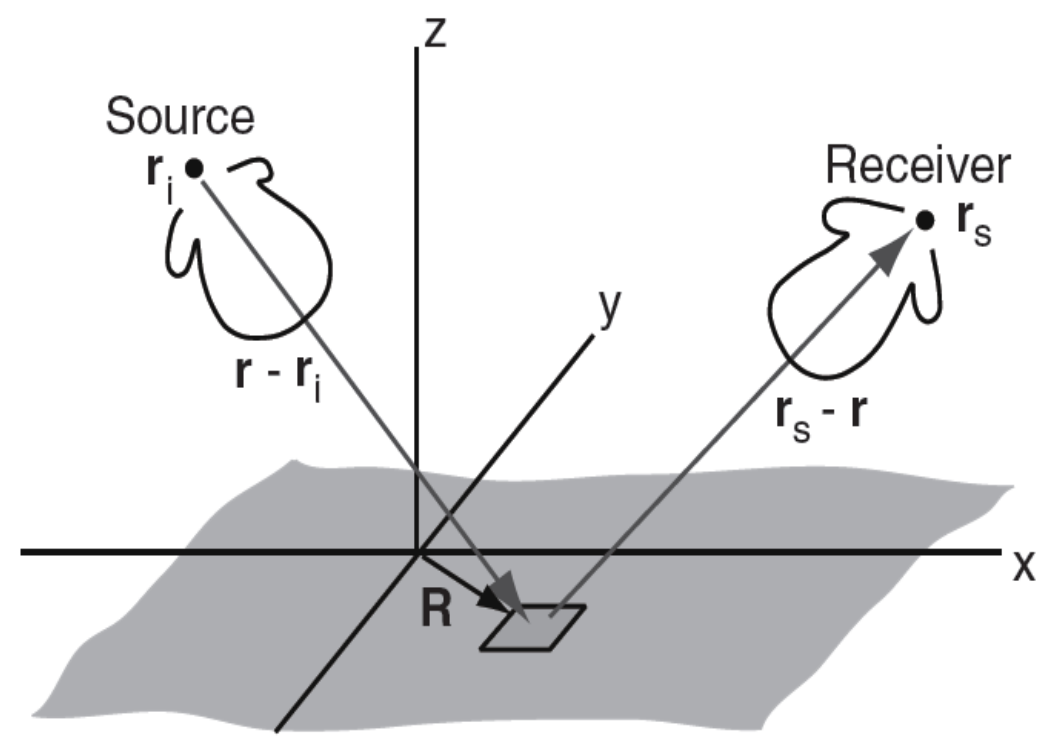

Figure B.2: Geometry for computing the mean square received voltage in bistatic scattering scenario. Note that $\mathbf{r}=(\mathbf{R}, 0)$ 
The generalization of eq. (2.24) is simply an integration that sums the contribution of infinitesimal patches of area to obtain the mean-square received voltage,

$$
<\left|V_{r}(t)\right|^{2}>=s_{r}^{2} \int\left|P_{i}\right|^{2} \sigma \frac{e^{-2 k_{w}^{\prime \prime}\left|\mathbf{r}_{s}-\mathbf{r}\right|}}{\left|\mathbf{r}_{s}-\mathbf{r}\right|^{2}}\left|b_{r}\left(\theta_{r}, \phi_{r}\right)\right|^{2} d^{2} R
$$

where

$$
R S=20 \log _{10} s_{r}
$$

is the receiver sensitivity giving the voltage per unit pressure and $b_{r}\left(\theta_{r}, \phi_{r}\right)$ is receiver directivity pattern see appendix $\mathrm{F}$ of reference [3]. $\theta$ and $\phi$ are grazing and azimuthal angles respectively, and subscript $r$ denotes receiver quantities.

The incoherent sum implied by eq. (B.18) is justified in most cases of interest. All the factors inside integrand of eq. (B.18) depend on the integration coordinates $\mathbf{R}=$ $(x, y)$ with $d^{2} R=d x d y$. The integration limits of eq. B.18) depend on the length of transmitted pulse and on the time $t$ at which the receiver voltage is evaluated. The timerange relation is

$$
\left|\mathbf{r}-\mathbf{r}_{i}\right|+\left|\mathbf{r}-\mathbf{r}_{s}\right|=c_{w} t_{0}(\mathbf{R})
$$

where $\mathbf{r}=(\mathbf{R}, 0)$ is the distance vector from origin to scattering region (point) $\mathbf{R}$ and $t_{0}(\mathbf{R})$ is the time required for acoustic energy to travel the total distance from source to the scattering patch an then to the receiver. This time varies with the position $\mathbf{R}$ of the scattering patch. In the simplest case, if the transmitted pulse has a rectangular envelope 
of length $\tau$, the limits of integration can be set by constraining $(x, y)$ such that

$$
t-\frac{\tau}{2}<t_{0}(\mathbf{R})<t+\frac{\operatorname{tau}}{2}
$$

with $t$ is the time at which one wishes to estimate the scattering mean-square pressure. Evaluation of eq. (B.18) is simple for the case in which $\mathbf{r}_{\mathbf{i}}=\mathbf{r}_{\mathbf{s}}$. The range limits becomes

$$
r_{t}-\frac{c_{w} \tau}{4}<\left|\mathbf{r}-\mathbf{r}_{i}\right|<r_{t}+\frac{c_{w} \tau}{4}
$$

where

$$
r_{t}=\frac{c_{w} t}{2}
$$

is the range associated with time of interest. Inequality B.22 defines the range limits on the ensonified region. 


\section{Appendix C}

\section{The volume scattering approximation}

The basic fluid sediment volume scattering model was developed by Stockhausen [24] with the key assumptions that

1. Volume scattering is sufficiently weak that the volume scattered acoustic field in the sediment is much smaller than incident field.

2. Sediment volume scattering can be characterized by a cross section that increases linearly with the ensonified volume.

3. Attenuation in the sediment limits the penetration to the depth that are much smaller than the distance from the source and receiver to scattering area.

4. The scattering effect of sediment -water is negligible.

With these assumption, the sediment volume scattering at high-frequency can be treated as an interface process and can be characterized experimentally and theoretically that same way as scattering by seafloor roughness was described. Further with an assumption that the source is unit, the equivalent model for volume scattering cross section is

$$
\sigma_{v o l}=\frac{\left|V_{w p}\left(\theta_{i}\right)\right|^{2}\left|V_{w p}\left(\theta_{s}\right)\right|^{2}}{|\rho|^{2}} \int_{-\infty}^{0} \sigma_{v}(z) e^{2 k_{w} \operatorname{Im}\left[P\left(\theta_{i}\right)+P\left(\theta_{s}\right)\right] z} d z
$$


where,

$$
P(\theta)=\sqrt{\left(v^{-2}-\cos ^{2} \theta\right)}
$$

and

$$
\left|V_{w p}\right|=\left|V_{w w}\right|+1
$$

with $v$ being the complex compressional wave speed/ water sound speed ratio, $i$ and $s$ subscripts denote for incidence and scattering. If the volume scattering was assumed being independent on the depth, the integral can be performed resulting in,

$$
\sigma_{v o l}=\frac{\left|V_{w p}\left(\theta_{i}\right)\right|^{2}\left|V_{w p}\left(\theta_{s}\right)\right|^{2} \sigma_{v}}{2 k_{w}|\rho|^{2} \operatorname{Im}\left[P\left(\theta_{i}\right)+P\left(\theta_{s}\right)\right]}
$$

where, $k_{w}$ is the water wavenumber. Equation (C.4) forms the basic for several sediment volume scattering models, which differ only in the assumption used to obtain the volume scattering cross section. The following dimensionless parameter, so-called normalized volume scattering parameter is use to quantify sediment volume scattering,

$$
\sigma_{2}=\frac{\sigma_{v}}{\alpha_{p}}
$$

where $\alpha_{p}$ is the attenuation in $\mathrm{dB} \mathrm{m}^{-1}$. 


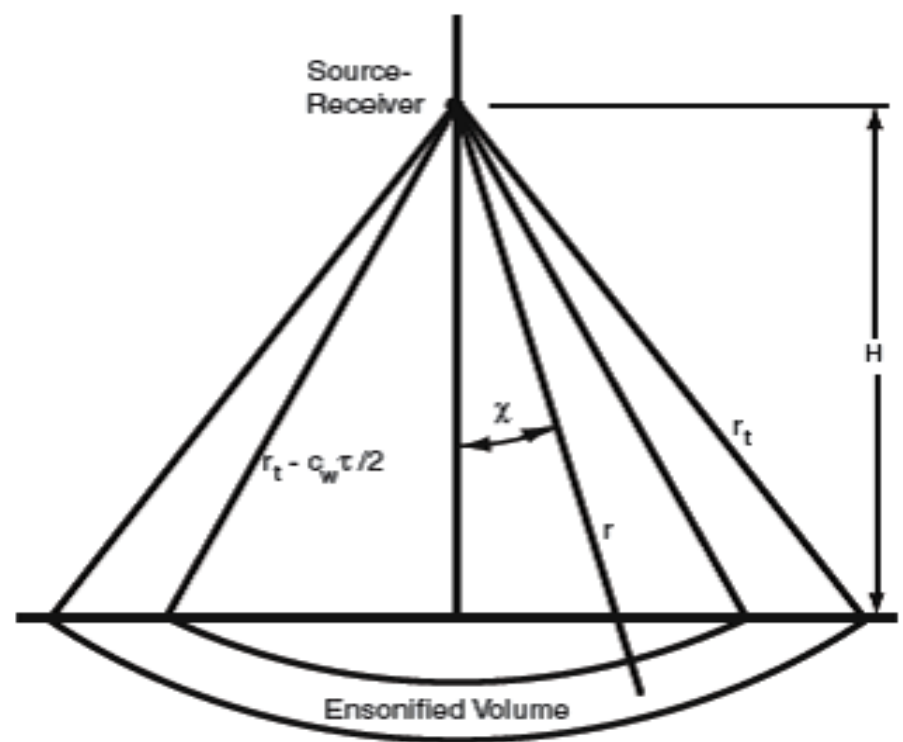

Figure C.1: Illustration of volume scattering geometry

For volume scattering, one must integrate over the ensonified volume as shown in Fig.C.1. To simplify the problem the interface is assumed flat and ray bending is ignored. This is reasonable assumption for the signal near vertical incidence. 